Michael Beißwenger (Dortmund)

\title{
Chattern unter die Finger geschaut: Formulieren und Revidieren bei der schriftlichen Verbalisierung in synchroner internetbasierter Kommunikation
}

Gliederung:

1. Einleitung

2. Grundbedingungen chatbasierter Kommunikation

3. Textrevisionen beim Chatten: Befunde auf Basis multimodaler Beobachtungsdaten

3.1 Durch Partnerbeiträge (,extrinsisch“) motivierte Revisionen

3.2 Nicht durch Partnerbeiträge (,intrinsisch“) motivierte Revisionen

3.2.1 Syntaktische Revisionen

3.2.2 Lexikalische Revisionen

3.2.3 Korrektur von Tippfehlern

3.2.4 Orthographische Revisionen

3.2.5 Morphologische Revisionen

3.2.6 Revision zum Zweck der Adressierung

3.2.7 Rein mentale Umplanungsaktivität

3.2.8 Gegenstandsbedingte Revisionen

3.2.9 Graphische Hervorhebung

4. Bewertung der Ergebnisse und Ausblick

\section{Einleitung}

Ähnlich wie die Prozessperspektive auf Texte (Schreiben) wichtige neue Impulse für die Textlinguistik liefern konnte, kann die linguistische Erforschung internetbasierter Kommunikation dadurch gewinnen, dass sie nicht nur die zwischen den Kommunizierenden ausgetauschten Produkte (die sprachliche Gestalt von E-Mail-Nachrichten, Foren- oder Chat-Beiträgen, Text Messages, Weblog-Einträgen), sondern auch die Prozesse ihrer sprachlichen Enkodierung in den Blick nimmt. Während im Falle mündlicher Kommunikation der Prozesscharakter der sprachlichen Äußerung bereits im Kommunikationsereignis selbst manifest wird, ist in schriftlicher Kommunikation (bei der in der Regel nur fertige Produkte ausgetauscht werden) die Prozessualität der sprachlichen 
Hervorbringung ein Datum, das nicht interaktiv, sondern lediglich für den Produzierenden selbst relevant ist. Dennoch können die Einsichtnahme in den Prozess der Entstehung schriftlicher Äußerungen und die Analyse von Vor- und Zwischenversionen, die nie „Produktreife“ erlangen, wichtige Aufschlüsse darüber liefern, wie die Schreiberinnen und Schreiber in einem bestimmten situationellen Kontext mit den Anforderungen einer bestimmten kommunikativen Aufgabe unter den Bedingungen einer bestimmten Kommunikationsform (z.B. Brief-, Chat-, E-Mail-Kommunikation) und mit Blick auf ein jeweils spezifisches individuelles Sprachhandlungsziel umgehen.

Von besonderem Interesse ist die Prozessperspektive für die Beschäftigung mit Kommunikationsformen, die sich für die Enkodierung von Kommunikaten des schriftlichen Mediums bedienen und zugleich aufgrund ihres dialogischen Charakters sowie ihrer Synchronizität mündlichen Formen des sprachlichen Austauschs nahe stehen. Solche Formen - einerseits der Chat, andererseits das Instant Messaging - sind erst mit dem Internet aufgekommen und gelten unter linguistischem Gesichtspunkt als innovativ:

Die mediengeschichtliche Novität des Chat besteht [...] darin, dass zum ersten Mal im großen Stil die Schrift zur dialogischen, synchronen Kommunikation im Distanzbereich verwendet wird (Storrer 2001: 439).

(Nicht von ungefähr hat die Chat-Kommunikation in den letzten eineinhalb Jahrzehnten ein zunehmendes Interesse der Linguistik auf sich gezogen. Koch/Oesterreicher bezeichnen in einer neueren Arbeit den Chat als ,eines der schönsten Beispiele dafür, daß im graphischen Medium eine relative, natürlich immer limitierte Annäherung an dialogische, spontane Nähesprachlichkeit möglich ist" (Koch/Oesterreicher 2007: 359). ${ }^{1}$

Gerade die mediale Limitiertheit der Annäherung an ,Nähe' ist für den Chat im Detail noch wenig erforscht. Dass die ChatKommunikation dem mündlichen Gespräch näher steht als dem monologischen Text, dürfte weitgehend unstrittig sein. Die Frage, wie sich der Chat im Detail zum Gespräch verhält und wie sich die Verwendung des graphischen Mediums auf die in ihm gegebenen kommunikativen Rahmenbedingungen auswirkt, wird in der Literatur hingegen z.T. un-

1 Die Konzepte, graphisch` bzw. ,medial schriftlich“ und ,phonisch` bzw. ,medial mündlich` werden in diesem Beitrag i.S.v. Koch/Oesterreicher $(1985 ; 1994 ; 2007)$ verwendet. Das ,Medium“ beschreibt nach dieser Konzeption die materiale Ebene kommunikativer Äußerungen, die entweder von lautlicher (= ,phonischer') Qualität ist oder sich der Schrift bedient und damit, graphische`Qualität aufweist. Tertium non datur. 
terschiedlich beantwortet. Das graphische Medium ist zunächst das klassische Medium für die Bewältigung der Bedingungen von Distanzkommunikation: „Die Schrift qua graphisches Medium ist nicht notwendige Bedingung - wenn auch ideales Instrument - zur Realisierung der kommunikativen Anforderungen der Distanz" (Koch/Oesterreicher 1994: 589).

Die grundsätzliche Frage ist, wie fundamental die Eigenschaften des Mediums die Bedingungen des Austauschs determinieren. Koch/ Oesterreicher selbst sprechen davon, dass das graphische Medium ,eine nicht zu unterschätzende ,bremsende " Wirkung bezüglich der Nähesprachlichkeit" habe (Koch/Oesterreicher 2007: 359). Ágel/Hennig weisen darauf hin, dass „bei der Etikettierung des Chat als ,konzeptionell mündlich“" der Einfluss der medialen Schriftlichkeit ,vielfach unterschätzt" (2007: 209) werde.

Hoffmann (2004) zieht für die Charakterisierung des Chat das ,Diskurs'-Konzept von Konrad Ehlich heran (vgl. z.B. Ehlich 1983; 1984; 1994; Ehlich/Rehbein 1986): Chat ist, ebenso wie die elementare Diskurskonstellation (face-to-face), auf eine aktuelle, dialogisch ausgerichtete Problembearbeitung im zeitlichen Nahbereich gerichtet. Hierbei ist die längerfristige Fixierung des einzelnen Kommunikats weniger wichtig als das Ergebnis der Interaktion, das ein gemeinsames Produkt sämtlicher Beteiligter darstellt. Charakteristisch für Chat ist nun gerade, dass er zur Prozessierung synchronen und dialogischen Austauschs die Überlieferungsqualitäten von Textformen nutzt, wodurch die ausgetauschten Beiträge erst in der Rezeption (also in der je individuellen Verarbeitung) diskursive Qualität gewinnen:

Das diskursive Moment besteht darin, dass der Chat anders als der Text nicht primär auf „die Bearbeitung des Vergessen““ (Ehlich 1989: 91) zielt, sondern auf aktuelle Verständigung. Was jeweils auf den Schirmen entsteht, erhält in der Rezeption diskursive Qualität. Zweck ist eine unmittelbare Kommunikation, die wechselseitige Verständigung aktualgenetisch, im zeitlichen Nahbereich anstrebt (Hoffmann 2004: 105).

Durch den Rückgriff auf Textformen erhalten die ausgetauschten Kommunikate gegenüber mündlich realisierten Diskursen eine andere sensorische Qualität: Koch/Oesterreicher (2007: 359) sprechen vom „,visuellen Prinzip der Graphie“ gegenüber dem „akustischen Prinzip der Phonie“. Weiterhin liegen graphische gegenüber phonisch realisierten Äußerungen, wenn auch nur kurzzeitig, als fixierte, extern manifeste Verhaltensspuren vor und werden so einer erneuten Bearbeitung zugänglich. Während die Phonie irreversibel und flüchtig ist, ist die Graphie reversibel und persistent und liefert 
einen „Außenspeicher“ für Inhalte und kognitive Prozesse; sie erlaubt es nicht nur, das, was uns momentan im Kopf steht, nach außen zu tragen - dies tut jede sprachliche Äußerung - sondern es außen aufzubewahren (Klein 1985: 10).

Eine spannende Frage in Hinblick auf eine präzise Profilierung der Chat-Kommunikation im Spannungsfeld von kommunikativer ,Nähe“ und ,Distanz' ist nun gerade die, ob die Nutzer chatbasierter Kommunikationsangebote bei der Produktion ihrer schriftlichen Beiträge von den durch die „Außenspeicherung“ gegebenen Möglichkeiten Gebrauch machen oder ob bei der Versprachlichung von Kommunikationsbeiträgen im Chat das graphische Medium (in Ermangelung einer freien Wahl zwischen Phonie und Graphie) in einer Weise genutzt wird, die der Verbalisierung im phonischen Medium nahe steht. In der gesprochenen Sprache ist die Hervorbringung unbedingter Linearität unterworfen: Nichts, was einmal als Segment artikuliert wurde, kann nachträglich wieder getilgt oder verändert werden. Einmal Geäußertes kann lediglich „,repariert“" werden, die mit einer Reparatur intendierte nachträgliche Rekonstruktion muss aber mental vom Hörer nachvollzogen werden und macht die Tatsache, dass ein repariertes Element geäußert wurde, nicht ungeschehen. Graphizität hingegen - insbesondere wenn sie, in prototypischer monologischer Textproduktion wie auch im Chat, mit einer Privatheit des Produktionsprozesses einhergeht - erlaubt die nachträgliche materiale Bearbeitung der Äußerung (die, solange sie noch nicht an ihre Adressaten übergeben wurde, Entwurfscharakter hat). Sie ermöglicht echte Revision, die für die späteren Rezipienten idealerweise spurlos bleibt:

Spoken language is permanent, whereas written is temporary. [...] Spoken language is permanent because once something is said, its impact cannot be erased, but something written can be crossed out, and it is as though it never was written (Frank Smith, zit. n. Tannen 1984: 29).

Lemnitzer/Naumann (2001) haben an Beispielen aus einem Mitschnittskorpus zu einem chatbasierten Seminar gezeigt, dass Konstruktionsbrüche, die für die gesprochene Sprache als Anakoluth beschrieben werden (vgl. Zifonun/Hoffmann/Strecker 1997: Kap. C 4.4) und dort der ,On line'-Reparatur von „Diskrepanzen zwischen Sprecherplan, Bedingungen für die Verwendung sprachlicher Mittel und Verbalisierung“ (Zifonun/Hoffmann/Strecker 1997: 445) dienen, durchaus auch in (verschickten) Chat-Beiträgen vorzufinden sind:

Beispiele 1-3: Ausschnitte aus dem Mitschnitt eines chatbasierten Seminars (Beispiele 8, 9 und 5 aus Lemnitzer/Naumann (2001: 479f:); Markierungen $M B)$ : 
(1) KERSTIN: Es ist daher guter Stil, bei Suchmaschinen oder Wörterbüchern mit Suchabfragen eine virtuelle Tastatur bereitzustellen... (Fri 1:56pm)

KERSTIN: auf der die nicth (Fri 1:56pm)

KERSTIN: die_nicht_vorkommenenden_ Zeichenen angeklickt werden können. (Fri 1:56pm)

(2) HUGO: Als nächstes war wichtig COMPASS 'beizububringen'___ob_das_angefragte

HUGO: bzzw _ zu erkennen, ob das angefragte Wort ein einem bestimmten Kontext vorkommt (Mon 5:34pm)

(3) Отто: Demnach beginnen Chunks mit einem Funktionswort und enden mit dem sog. d-major headf (Mon 4:4lpm)

Отто: (ohne f) (Mon 4:41 pm)

Die Beispiele von Lemnitzer/Naumann legen nahe, dass zentrale reparative Verfahren aus der Mündlichkeit unter bestimmten Umständen in der Schriftlichkeit des Chat nachgebildet werden. Allerdings fällt auf, dass die Reparatur in den von Lemnitzer/Naumann als Beispiel für Retraktion gegebenen Belegen durchweg an der Grenze zweier vom betreffenden Chatter nacheinander verschickter Beiträge auftritt: ${ }^{2}$ In Beispiel (1) liefert KERSTIN in einem in derselben Minute nachgeschobenen dritten Beitrag ein Reparationsangebot für den in ihrem zweiten Beitrag aufgetretenen Tippfehler nicth nach. Ganz offensichtlich hat sie diesen erst nach der Verschickung ihres Vorbeitrags (durch Nachlesen) entdeckt; da der Beitrag bereits verschickt ist, ist eine Revision vor der Herausgabe nicht mehr möglich. Folglich hat sie nur die Möglichkeit, den Fehler entweder zu ignorieren oder ihn nachträglich zu berichtigen, indem sie dem für defizient befundenen Ausdruck eine dafür einzusetzende optimierte Alternative (die nicht) nachfolgen lässt, wobei sie die begonnene Konstruktion ab der letzten Phrasengrenze wiederholt. In Beispiel (2) integriert HUGO nachträglich - ebenfalls, weil sein Vorbei-

2 Längere Kommunikationsbeiträge auf mehrere Textbeiträge zu verteilen und diese sukzessive zu verschicken (,Splitting') war Teil des Seminarsettings und wurde von den beteiligten Studierenden über eine "Chatikette“ mit Regeln für die Teilnahme an den Seminarchats explizit eingefordert:

„Wenn der Beitrag länger wird, bitte schon mal einen Teil (eine Zeile) losschicken, die Zeile dann bitte mit ,... “ beenden. Die anderen wissen dann: es kommt noch was von Ihnen. Wenn Sie eine Zeile sehen, die mit drei Punkten endet: Haben Sie Geduld, bis der/die SchreiberIn fertig ist. Bitte erst dann antworten [durch diese Regel wird zweierlei erreicht: erstens werden die lesenden Teilnehmer nicht durch eine mehrzeilige Message zu lange gebunden; zweitens erhalten so auch langsamere SchreiberInnen eine bessere Chance zur Teilnahme. ...]“ (Lemnitzer \& Naumann 2001: 475). 
trag bereits verschickt ist und daher nicht mehr entsprechend umgearbeitet werden kann - in seinem zweiten Beitrag eine Ergänzung zu der Struktur, die er in seinem vorigen Beitrag eröffnet hat und wiederholt den letzten Teil des Vorbeitrags ab derjenigen Stelle, an welcher die Ergänzung einzufügen ist. Auch hier ergibt sich ein Konstruktionsbruch, der dadurch aufgelöst werden kann, dass der Rezipient die Bruchstelle, den zu ersetzenden und den ersetzenden Ausdruck durch syntaktische Interpretation identifiziert und dann in seinem Arbeitsgedächtnis den zu ersetzenden Ausdruck aus HugOs Äußerung mit dem ersetzenden Ausdruck überschreibt. In Beispiel (3) wird die Reparatur auch hier geht es wieder um einen Tippfehler - explizit sprachlich markiert und zugleich mit der Benennung des unbeabsichtigt eingegebenen Graphems eine Suchanweisung in Bezug auf den zu ersetzenden Ausdruck formuliert.

Fälle wie die von Lemnitzer/Naumann beschriebenen zeigen tatsächlich eine gewisse Ähnlichkeit zu Reparaturverfahren in gesprochener Sprache, wenngleich in den angeführten Beispielen auf unterschiedliche Weise repariert wird bzw. die Diskrepanzen zwischen Planung und Verbalisierung auf unterschiedlichen sprachlichen Ebenen anzusiedeln sind und mit unterschiedlichen Mitteln bearbeitet werden (Änderung der Projektionsstruktur in Beispiel 2, metasprachliche Tippfehlerkorrektur in Beispiel 3). Inwiefern hier Reparaturverfahren einfach in die Graphie übertragen werden bzw. ob es sich bei den beschriebenen Fällen eher um eine funktionale Nachbildung der Reparatur unter den Bedingungen medialer Schriftlichkeit handelt, soll an dieser Stelle nicht diskutiert werden.

Der vorliegende Beitrag untersucht Fälle der nachträglichen Veränderung eingegebener Texte beim Chatten auf der Ebene ihrer Hervorbringung. Während die Besonderheiten an der sprachlichen Oberfläche von via Chat ausgetauschten Textbeiträgen in der Forschung bereits recht gut beschrieben sind, ${ }^{3}$ ist der Entstehungsprozess von ChatBeiträgen nach wie vor weitgehend unerforscht. Der Grund hierfür liegt vermutlich weniger in einer einseitigen Forschungsperspektive auf den Gegenstand als vielmehr darin, dass aufgrund der zeitlichen Umstände der schriftlichen Beitragsproduktion zunächst davon auszugehen ist, dass Kommunikationsbeiträge so schnell als möglich eingetippt und dann unmittelbar verschickt werden, dass also die Beitragseingabe im Grunde ähnlich linear verläuft wie die Äußerungsproduktion in pho-

3 Für einen Überblick vgl. z.B. Runkehl/Schlobinski/Siever (1998), Crystal (2001), Hennig (2001), Storrer (2001; 2009 i.Dr.) sowie die Beiträge in Beißwenger (2001). 
nisch realisierter Kommunikation. Ein weiterer, forschungspraktischer Grund hat damit $\mathrm{zu}$ tun, dass Mitschnitte (= Speicherinstanzen aller über den Chat-Server verschickten sprachlichen Beitragsprodukte) vergleichsweise einfach und in großer Zahl erhoben werden können, die Dokumentation der Prozessualität der Textproduktion am Bildschirm hingegen mit einem hohen Aufwand verbunden ist, sowohl was die Gewinnung als auch was die Aufbereitung der Daten für Analysezwecke anbelangt.

Ich werde im Folgenden zunächst einige Grundbedingungen chatbasierter Kommunikation skizzieren, die für eine Charakterisierung der spezifischen Versprachlichungs- und Äußerungsbedingungen beim Chatten zentral sind (Abschnitt 2). Anschließend werde ich die Ergebnisse zweier Untersuchungen zu Textrevisionen bei der Produktion von Chat-Beiträgen vorstellen, die ich auf der Basis eines Korpus mit multimodalen Daten zu Nutzeraktivitäten beim Chatten durchgeführt habe (Abschnitt 3). Abschließend werden die Ergebnisse der Analysen in Bezug auf ihre Relevanz für die Verortung des Chat zwischen ,Nähe“ und ,Distanz' bewertet.

\section{Grundbedingungen chatbasierter Kommunikation}

Chat-Kommunikation ist dialogische Kommunikation: Sie leistet eine kommunikative Problembearbeitung im zeitlichen Nahbereich über die Ausführung kooperativer Handlungsschritte durch zwei oder mehrere Kommunikanten. Die materiale Enkodierung von Kommunikaten ist dabei auf das graphische Medium festgelegt. Das von der Technologie vorgegebene Modell des Kommunikationsprozesses ist nicht den Produktions- und Verarbeitungsbedingungen synchroner mündlicher Kommunikation nachempfunden, sondern dem Austausch von Kommunikaten in zeitversetzter Distanzkommunikation: Äußerungen werden nicht zur Laufzeit ihrer Hervorbringung übermittelt und als kontinuierliches, flüchtiges Signal verarbeitet, sondern in Form von sprachlichen Produkten zu produzentenseitig bestimmten Zeitpunkten an die Adressaten übergeben (Prinzip der en bloc-Verschickung).

Dennoch erfordert chatbasierter Austausch eine synchrone Orientiertheit aller Beteiligten auf die Teilhabe am Kommunikationsprozess. Die Beteiligten können dabei beliebig zwischen produktivem und rezeptivem Teilhabemodus wechseln, die parallele Ausübung von Aktivitäten gleichen Typs (z.B. gleichzeitiges Produzieren zweier oder mehrerer Nutzer) wird dabei üblicherweise vom System nicht kontrolliert oder eingeschränkt. Produzierte Beiträge werden nach ihrer Verschi- 
ckung vom Server in der Reihenfolge ihrer Entgegennahme weiterübermittelt. Die Linearisierung von Beiträgen ist somit - gegenüber dem Gespräch - der intersubjektiven Aushandlung durch die Kommunizierenden weitgehend entzogen und wird statt dessen von einer technischen Instanz generiert, für die Kohärenz- oder Sequenzkriterien keine Rolle spielen. Die lokale Aushandlung einer Rollenkonstellation (im Sinne des ,one party talks at a time“-Prinzips natürlicher Gespräche) bzw. einer kohärenten Beitragsabfolge zwischen den Kommunizierenden ist daher bestenfalls metakommunikativ (mit Einschränkungen), nicht aber zur Laufzeit möglich.

Für die Produktion und Rezeption von Kommunikationsbeiträgen, die Koordination zwischen den Beteiligten und die Adaption der individuellen Handlungsplanung an den Stand des Kommunikationsverlaufs sind insbesondere die folgenden Bedingungen prägend: ${ }^{4}$

\section{Graphische Enkodierung:}

Kommunikate sind im graphischen Medium zu enkodieren. Dies gilt sowohl für die Chat-Technologie als auch für alle anderen ,klassischen" internetbasierten Kommunikationstechnologien und die durch sie konstituierten Kommunikationsformen (E-Mail, Mailinglisten, Newsgroups und MUDs sowie - als speziell WWW-basierte Formen Homepages, Gästebücher, Foren und Webchats) und hat technologiegeschichtliche Gründe: $\mathrm{Zu}$ der Zeit, zu der die Vorläufer heutiger Chatund Messaging-Systeme und schließlich mit dem INTERNET RELAY CHAT (IRC) der Prototyp heutiger synchroner internetbasierter Kommunikation entwickelt wurde(n), lag eine zeitnahe Übermittlung von Audiodaten in Computernetzen aufgrund der geringen Übertragungsraten nicht im Bereich des Realisierbaren; als Medium für die materiale Enkodierung sprachlicher Information musste daher pauschal das graphische Medium (beschränkt auf das im ASCII-Zeichensatz $z^{5}$ enthaltene Zeicheninventar) herangezogen werden. Die Tatsache, dass Äußerungen, die für den Austausch via Chat bestimmt sind, graphisch enkodiert

4 Für eine Gegenüberstellung der Grundbedingungen prototypischer mündlicher Kommunikation mit denen der Chat-Kommunikation vgl. z. B. auch Hennig (2001: 222235) und Hoffmann (2004: 103-106). Die Auswirkungen der technologischen Rahmenbedingungen auf chatbasierte Kommunikationsprozesse sind im Detail in Beißwenger (2007) beschrieben und diskutiert.

5 Das Akronym ASCII steht für American Standard Code for Information Interchange und bezeichnet cum grano salis dasjenige Inventar an (für das Englische relevanten) lateinischen Schriftzeichen, arabischen Ziffernzeichen und Sonderzeichen, die sich mittels einer handelsüblichen Tastatur erzeugen lassen. 
werden müssen, ist somit auf infrastrukturelle Rahmenbedingungen zurückzuführen und nicht etwa darauf; dass die Schrift den Entwicklern der ersten Chat-Systeme als in besonderer Weise für synchronen Austausch geeignet erschienen wäre.

Der Wechsel vom phonischen auf das graphische Realisierungsmedium bedeutet für die Realisierung synchroner Distanzkommunikation nicht lediglich, dass schriftlich enkodierte Beiträge eine zu mündlichen unterschiedliche materiale Qualität aufweisen. Mit Graphizität (auch wenn diese - etwa auf temporären digitalen Trägern oder auf einer beschlagenen Fensterscheibe - nur vorübergehend vorgehalten wird) einher geht eine wenigstens kurzzeitige Fixierung, wodurch der sprachlichen Äußerung über den Moment ihrer Hervorbringung hinaus Persistenz verliehen wird. Graphisch enkodierte Äußerungen können somit - zumindest für die Dauer ihrer Fixierung - nicht nur einmalig, sondern bei Bedarf mehrfach rezipiert werden. Durch Nutzung der Bearbeitungs- und Speicherfunktionen des Computers können digital fixierte graphische Äußerungen darüber hinaus nachträglich modifiziert und im Wortlaut vervielfältigt werden.

\section{En bloc-Übermittlung von Kommunikaten:}

Die Chat-Technologie modelliert den Kommunikationsprozess als eine Abfolge von Schritten, die konsekutiv aufeinanderfolgen. Die Hervorbringung eines Beitrags durch den Produzenten (die sprachliche Enkodierung) wird dabei von dessen Veräußerung (Herausgabe an die übermittelnde Instanz und Übermittlung an die Adressaten) separiert. Dies zeigt sich daran, dass die Veräußerung vom Produzenten (durch Ausführung einer Verschickungsanweisung, z.B. Betätigung der Eingabetaste oder Betätigung einer entsprechenden Schaltfläche mit der Maus) jeweils explizit veranlasst werden muss. Da es sehr unökonomisch ist, nach jedem auf der Tastatur eingegebenen Zeichen die benötigte Verschickungshandlung auszuführen, sind die minimalen veräußerten Einheiten in der Chat-Kommunikation in aller Regel nicht Einzelzeichen, sondern Zeichensequenzen. Im Normalfall wird der komplette Beitrag, den ein Beteiligter $\mathrm{zu}$ einem bestimmten Zeitpunkt $\mathrm{zu}$ leisten beschließt, erst vollständig enkodiert und dann als Ganzer verschickt. Bisweilen wird mit dem sog. „Splitting“ eine Strategie angewandt, mit der bereits vor Abschluss der Enkodierung des vollständigen intendierten Kommunikationsbeitrags Verschickungshandlungen ausgeführt werden. Bei den Einheiten, die in diesem Falle verschickt werden, handelt es sich aber in aller Regel ebenfalls bereits um Zeichenfolgen und nicht um Einzelzeichen. Die Schriftzeichen, die ein Chat-Nutzer produziert, werden somit nicht zur Laufzeit ihrer Hervorbringung übermittelt, 
sondern immer nur en bloc, d.h. immer nur dann, wenn der Produzent auch eine Verschickungshandlung ausführt. Ein inkrementelles Verfolgen des Versprachlichungsprozesses ist somit ausgeschlossen (vgl. Beißwenger 2003: 204 und 209f.; Bittner 2003: 196; Dürscheid 2004: 151).

Das konsekutiv konzipierte Modell des Kommunikationsprozesses entspricht insgesamt eher einem klassischen Publikationsprozess als der Einheit von Versprachlichung, Veräußerung, Wahrnehmung und rezeptiver Verarbeitung in mündlich realisierter synchroner Kommunikation. Das darin vorgesehene en bloc-Prinzip hat weitreichende Konsequenzen: Dadurch, dass eine eingegebene Zeichenfolge, so lange sie noch nicht verschickt ist, nur ,privat" im Eingabeformularfeld auf dem Bildschirm des Produzenten vorgehalten wird, kann sie prinzipiell so lange und so oft bearbeitet werden, bis der Produzent sie für publikationsreif befindet und per Verschickungsanweisung (vergleichbar einer Imprimatur) willentlich herausgibt. Nachträgliche Modifikationen einmal eingegebener Zeichensequenzen sind möglich - und kommen vor (siehe Abschnitt 3).

3. Visuelle Qualität des graphischen Mediums:

Während durch das en bloc-Prinzip der Prozess der Hervorbringung und der Akt der Veräußerung (Verschickung) der sprachlichen Äußerung als einander strikt nachgeordnete Teile des Kommunikationsprozesses modelliert und damit zeitlich voneinander entkoppelt werden, ergibt sich durch die visuelle Qualität des graphischen Mediums zudem eine zeitliche Entkopplung der Übermittlung des verschickten Kommunikats von dessen Wahrnehmung und mentaler Verarbeitung durch den oder die Adressaten: Ergebnisse graphischer Enkodierung können nur visuell perzipiert werden, sind somit reine Augenmedien und ,für die Ohren unsichtbar". Mit Phonizität einher geht hingegen die Unmittelbarkeit der Übertragung (mündlich Geäußertes dringt - kraft seiner materialen Qualität als Schallereignis - unweigerlich ans Ohr). Die visuelle Qualität des graphischen Mediums macht es daher erforderlich, dass der Adressat, um neue Kommunikationsbeiträge wahrzunehmen, zunächst seine visuelle Aufmerksamkeit auf denjenigen Ort richten muss, von dem er weiß, dass dort üblicherweise für ihn bestimmte schriftliche Äußerungen dargestellt werden. So lange er dies nicht tut, ist ihm nicht einmal die Existenz der betreffenden Äußerung bekannt. 
Eine mündliche Äußerung wirklich zu überhören, ist hingegen bei normalen Umweltbedingungen so gut wie unmöglich. ${ }^{6}$

\section{Synchronizität ohne Synchronisierung:}

Die erfolgreiche Übermittlung von Kommunikaten an einen oder mehrere Adressaten sowie der Empfang von Kommunikaten anderer Urheber erfordert eine synchrone Kopräsenz der Kommunikationsbeteiligten: Die Beteiligten müssen zur selben Zeit in derselben Kommunikationsumgebung (einem sog. "Chatroom“ oder "ChatChannel“) angemeldet (,eingeloggt") sein. Je nachdem, ob in der betreffenden Kommunikationsumgebung (abhängig von den Merkmalen des verwendeten Chat-Systems) die ausgetauschten Beiträge über die gesamte „Sitzung“ (d.h. denjenigen Zeitraum, während dessen man als Nutzer in der Kommunikationsumgebung angemeldet ist) oder nur für eine gewisse Dauer auf dem Bildschirm vorgehalten werden, ist neben dem bloßen Angemeldetsein weiterhin eine zeitgleiche Orientierung der Beteiligten auf die Teilhabe an der Kommunikation erforderlich.

Synchronizität bedeutet in der Chat-Kommunikation zunächst einmal nichts weiter als die zeitgleiche kommunikative Verfügbarkeit der Kommunikationsbeteiligten in derselben Kommunikationsumgebung und nicht die vollständige Synchronisierung der Wahrnehmung(en) des Kommunikationsverlaufs seitens der einzelnen Beteiligten (im Sinne einer wechselseitigen Verarbeitung von Verhaltensäußerungen simultan zur Laufzeit ihrer Hervorbringung). Eine solche ist in der ChatKommunikation einerseits aufgrund des en bloc-Prinzips und des daraus resultierenden Ausschlusses des Prozesscharakters der Verbalisie-

6 Obwohl die Chat-Technologie die durch sie spezifizierte Kommunikationsform als synchronen Austausch konzipiert, lässt sich anhand von Nutzerbeobachtungen nachweisen, dass der Zeitpunkt der Anzeige eines Beitrags im Bildschirmverlaufsprotokoll und der Zeitpunkt seiner offiensichtlichen Wahmehmung durch den Adressaten nicht unerheblich divergieren können. Als Konsequenz ist davon auszugehen, dass auch die mentalen Repräsentationen der einzelnen Beteiligten darüber, was zu einem bestimmten Realzeitpunkt der aktuelle Stand des Kommunikationsverlaufs ist, u.U. erheblich von einander abweichen. In Beißwenger (2007: 473ff.) ist ein Beispiel dargestellt, das nahelegt, dass, bezogen auf die Anzeigezeitpunkte der zurückliegenden Beiträge auf dem Bildschirm, zu ein- und demselben Zeitpunkt die kognizierten Stände zweier ChatTeilnehmerinnen um ganze 47 Sekunden voneinander abweichen. Dies hat erhebliche Konsequenzen für die Handlungskoordination zwischen den Beteiligten. 
rung von der Übermittlung sowie andererseits aufgrund der visuellen Qualität graphischer Enkodierung ausgeschlossen.,8

\section{Autonome Wahl von Aktivitätstypen anstatt Aushandlung von Rollenkonstellationen zur Laufzeit:}

In Standard-Chat-Systemen gibt es keine exklusive Produzentenrolle, die der Sprecherrolle mündlicher Gespräche vergleichbar wäre. Stattdessen kann jeder Beteiligte jederzeit autonom und ohne dies mit den Partnern aushandeln zu müssen, von der Rezeption zur Produktion wechseln und die Enkodierung eines eigenen Beitrags aufnehmen. Die auf produktive Beteiligung am Kommunikationsgeschehen gerichteten Aktivitäten werden somit nicht linearisiert, sondern häufig parallel bzw. zeitlich überlappend ausgeübt, ohne dass der einzelne Chatter von den momentanen Aktivitäten seiner Partner weiß. Für die (künstliche) Linearisierung der Beiträge nach deren Verschickung sorgt die schon benannte Server-Routine:

7 Dies gilt übrigens auch für Messaging-Systeme wie UNIX TALK (Meise-Kuhn 1998), bei denen mit jedem Tastaturanschlag eine implizite Verschickungsanweisung ausgeführt und Zeichen um Zeichen direkt nach Eingabe übermittelt wird. Hier ist zwar die zeitliche Entkopplung von Produktion und Übermittlung aufgehoben und die Prozesshaftigkeit der Verbalisierung der Wahrnehmung durch die Kommunikationspartner prinzipiell zugänglich; dennoch wird aufgrund der visuellen Qualität des graphischen Mediums auch in solchen Systemen das Übermittelte nicht unmittelbar an den Wahrnehmungsapparat zugestellt, sondern muss zunächst mit den Augen „entdeckt“ werden. In synchroner mündlicher Kommunikation hingegen ist die unbedingte Simultaneität von Verbalisierung und $\mathrm{W}$ ahrnehmung zwingend.

8 Um die fundamentalen Unterschiede der kommunikativen Rahmenbedingungen des Chat gegenüber derjenigen synchroner mündlicher Kommunikation herausstellen zu können, erscheint es mir sinnvoll, Simultaneität (= die zeitliche Parallelität der Hervorbringung von Verhaltensäußerungen mit ihrer Wahmehmung/Verarbeitung durch die Partner) nicht als ein Merkmal von Synchronizität zu fassen, sondern als eigenes Merkmal für die Beschreibung von Kommunikationsformen zu behandeln. Synchronizität meint in dieser Konzeption lediglich die Gleichzeitigkeit des Orientiertseins der Kommunikanten auf den gemeinsamen Austausch. Ein solches Synchronizitätskonzept, das Simultaneität nicht zwangsläufig einschließt, erlaubt es, Gespräche als „synchron und simultan“, Chat hingegen als lediglich „synchron“ zu beschreiben und somit die bislang eher behelfsmäßigen Charakterisierungen des Chat als „quasi-synchron“" (z.B. Garcia/Jacobs 1998; Dürscheid 2005; 2006) oder „,annähernd synchron“ (z.B. Beißwenger 2002; 2003) zu vermeiden. Eine Diskussion unterschiedlicher Konzeptionen von Synchronizität und Simultaneität in der linguistischen Chat-Forschung findet sich in Spitzmüller (2005: 10f.) und in Beißwenger (2007: 35ff.). 
6. Linearisierung nach dem „Mühlen-Prinzip“:

Eine Routine auf dem Chat-Server sorgt dafür, dass eintreffende Teilnehmerbeiträge für die Darstellung am Bildschirm in eine lineare Abfolge gebracht werden. Standardmäßig werden Beiträge in der Reihenfolge ihres Eintreffens auf dem Server weiterübermittelt und im Bildschirmverlaufsprotokoll ${ }^{9}$ durch einen forcierten Absatzwechsel von einander separiert; ${ }^{10}$ gleichzeitig eintreffende Beiträge werden vor der Weiterübermittlung ebenfalls auf zwei Absätze verteilt. Kohärenzkriterien spielen bei dieser Form der technisch erzeugten Linearisierung keine Rolle.

Bei den skizzierten Charakteristika handelt es sich teilweise um Merkmale, die traditionell aus Formen direkter, mündlicher Kommunikation vertraut sind, teilweise um Merkmale, die als typisch gelten können für Formen der zeitversetzten Distanzkommunikation. Für Näheformen charakteristisch sind u.a.:

- die Synchronizität des Orientiertseins der Partner auf die Teilhabe am gemeinsamen Austausch;

- die dialogische Organisation des Austauschs mit der Möglichkeit, jederzeit vom Rezipieren zum Produzieren zu wechseln, wobei das Rezipierte jeweils unmittelbar zuvor vom Partner erzeugt wurde und das selbst Produzierte unmittelbar anschließend vom Partner rezipiert werden kann.

Für Distanzformen charakteristisch sind u.a.:

- die fehlende Synchronisierung (Nicht-Simultaneität) von Hervorbringung und Verarbeitung;

- die Verwendung der Schrift als Enkodierungsmedium;

9 Das Bildschirmverlaufsprotokoll ist die temporäre Protokollierung der zwischen den Kommunikanten ausgetauschten Textbeiträge auf dem Bildschirm eines Teilnehmerrechners. Der Kommunikationsverlauf wird vom System lediglich zu Zwecken einer direkten Verarbeitung durch die Kommunikanten vorgehalten; die hierzu erzeugte Protokolldatei steht dem Teilnehmer nach Beendigung seiner Chat-Teilnahme in der Regel nicht mehr zur Verfügung. Angezeigt wird vom Verlaufsprotokoll jeweils nur der jüngste Teil; bei den meisten Chat-Systemen lassen sich ältere Teile bei Bedarf jedoch wieder individuell in die Anzeige zurückholen (Scroll-Funktion).

10 Wichter (1991: 78f.) spricht diesbezüglich vom „Mühlen-Prinzip: Wer zuerst kommt, mahlt zuerst.“" 
- die en bloc-Modellierung der sprachlichen Äußerung, bei welcher die zeitliche Einheit von Hervorbringung und Übertragung aufgebrochen wird und der Verbalisierungsprozess $\mathrm{zu}$ einer privaten Leistung des Produzierenden wird, der vom Partner nicht eingesehen werden kann.

Aus der innovativen Kombination von Nähe- und Distanzmerkmalen und den daraus resultierenden Äußerungs-, Wahmehmungs- und Koordinationsbedingungen ergibt sich als Grundproblem der ChatTeilnahme eine Situation, die ich hier als das „Chatter-Dilemma“ bezeichnen möchte und die insbesondere von ungeübten Chattem häufig als Stress empfunden wird: Um in einer Kommunikationsform, die keine laufzeitbegleitenden Möglichkeiten zur Koordination zwischen den Beteiligten bereitstellt, möglichst optimal an geeigneten Punkten eigene Initiativen einbringen und kommunikative Züge anderer Beteiligter respondieren zu können, muss man idealerweise permanent gleichzeitig produzieren und rezipieren, was aber aufgrund der unterschiedlichen kognitiven Anforderungen, die sich bei der Verbalisierung und bei der Verarbeitung von sprachlicher Information stellen, bestenfalls kurzzeitig möglich ist. Folglich kann man die beiden Beteiligungsmodalitäten nur alternierend wahrnehmen.

Fasst ein Chat-Beteiligter an einem bestimmten Punkt des Kommunikationsverlaufs, z.B. nach der Rezeption eines Partnerbeitrags, den Plan, auf diesen Partnerbeitrag zu reagieren und daran mit einer bestimmten Folgehandlung anzuschließen, so muss er vom rezeptiven in den produktiven Teilhabemodus wechseln, um seinen Plan zu verbalisieren und anschließend das Verbalisierungsergebnis zu verschicken. Während er seinen Beitrag enkodiert, wird er vorwiegend mit dem Monitoring der eigenen Texteingabe (visuelle Kontrolle der motorischen Aktivitäten auf der Tastatur bzw. der entstehenden Zeichensequenz im Texteingabeformularfeld der Benutzeroberfläche) befasst sein und dem Bildschirmverlaufsprotokoll einen wesentlich kleineren Teil seiner Aufmerksamkeit widmen können, als wenn er im Rezeptionsmodus verblieben wäre. Dadurch steigt aber das Risiko, dass er zugunsten des Produzierens wichtige Neuerungen im Bildschirmverlaufsprotokoll registriert. Beispielsweise könnte ein neu im Verlaufsprotokoll eintreffender Partnerbeitrag seinen aktuell in Versprachlichung befindlichen Handlungsplan als komplett redundant oder thematisch nicht mehr ohne Weiteres anschließbar qualifizieren - wenn er von ihm Kenntnis nähme. Je länger er also mit Versprachlichung befasst ist, desto höher die Gefahr, dass der in Arbeit befindliche Beitrag nicht mehr ,passt“. Unter diesem Gesichtspunkt erscheint es also als opportun, die Phase zwi- 
schen dem Fassen des Handlungsplans und der Fertigstellung des sprachlichen Produkts so kompakt als möglich zu halten: Je kürzer die Produktionszeit, desto geringer die Chance, dass zwischenzeitlich ein anderer Beteiligter mit einem eigenen neuen Beitrag „querschieß $t^{\text {“" und }}$ den eigenen Plan ganz oder zu Teilen hinfällig macht.

Je intensiver sich der Beteiligte auf die Produktion konzentriert, um seinen geplanten Beitrag so zügig als möglich fertigzustellen, um so weniger Ressourcen wird er während des Produzierens auf die visuelle Kontrolle des Bildschirmverlaufsprotokolls verwenden können. Intensives Produzieren steigert somit ebenfalls das Risiko, etwas Wichtiges zu verpassen. Die Alternative besteht darin, während des Produzierens häufig innezuhalten und das Bildschirmverlaufsprotokoll zu überprüfen, um den in Versprachlichung befindlichen Handlungsplan ggf. frühzeitig entsprechend der aktuellen Weiterentwicklung des Protokolls modifizieren und an den aktuellen Stand anpassen zu können. Dies jedoch verlängert wiederum den Produktionsprozess als Ganzen und birgt im ungünstigen Fall (und insbesondere bei hoher Teilnehmerzahl) das Risiko, überhaupt keinen Beitrag abzuschließen und zum Zug zu kommen.

Wer selbst schon einmal gechattet hat, wird möglicherweise die Erfahrung gemacht haben, dass trotz des Produktionsdrucks die Produktion von Chat-Beiträgen nicht immer unbedingt ein linearer Enkodierungsprozess ist, sondern im Einzelfall diskontinuierlich verlaufen kann. Unterbrochen wird der Produktionsprozess z.B. dann, wenn während der Texteingabe ein neuer Partnerbeitrag im Verlaufsprotokoll bemerkt wird oder wenn sich während des Tippens eine Formulierung als nicht zielführend oder zu kompliziert erweist. In solchen Fällen kommt es nicht selten zu Überarbeitungen des eingegebenen Textes. Das, was letzten Endes als Prozessergebnis für die Partner sichtbar wird, ist somit nicht zwangsläufig (in allem) mit dem identisch, was im Zuge des Prozesses eingegeben wurde. Das, was bis zur Verschickung eines Beitrags an den Server im Eingabeformularfeld steht, muss nicht das Endergebnis, sondern kann eine Roh- oder Zwischenversion sein. Was am Ende eines Beitragsproduktionsprozesses als Produkt verschickt wird, ist bisweilen zu Beginn der Eingabeaktivität noch gar nicht bis ins sprachliche Detail vorkonzipiert.

Einsichten in die Besonderheiten des individuellen Umgangs mit dem „Chatter-Dilemma“ dürften für die Diskussion der Nähe/Distanz der Kommunikationsform wichtige neue Impulse liefern. Textrevisionen können in diesem Zusammenhang einen guten Einstiegspunkt bilden, da sie sich - im Gegensatz zu anderen, schwieriger beobachtbaren Phänomenen - direkt am sprachlichen Material manifestieren. 


\section{Textrevisionen beim Chatten: Befunde auf Basis multimodaler Beobachtungsdaten}

Im Folgenden beschreibe ich verschiedene Typen von Textrevisionen beim Chatten. Die einzelnen Typen wurden aus der Analyse von Beobachtungsdaten zur Prozessualität der Textproduktion beim Chatten entwickelt. Das zugrunde gelegte Korpus umfasst Transkripte zu Verhaltensäußerungen in verschiedenen (verbalen und nonverbalen) Modi, die an Probanden in einer Laborsituation während der Teilnahme an einem Beratungs-Chat beobachtet werden konnten. Die empirische Basis für die Transkripte bildeten zum einen Bewegtbild-Aufzeichnungen aller Bildschirminhalte und -aktivitäten, die während der ChatTeilnahme am Bildschirm der Probanden sichtbar waren. Zur Aufzeichnung wurde eine Screen Capturing-Software verwendet, die im Hintergrund auf dem Rechner lief und dabei automatisch eine Videoaufzeichnung dessen erzeugte, was am Bildschirm zu sehen war (inklusive Mausbewegungen und Tastatureingaben). Zum anderen wurden die 32 Probanden anhand einer Stativkamera frontal gefilmt, um ihre körperlichen Aktivitäten, insbesondere ihr Blickrichtungsverhalten, zu erfassen. Beide Aufzeichnungen wurden jeweils so dokumentiert, dass für jedes darin bezeugte Ereignis der Realzeitpunkt seines Auftretens rekonstruiert werden konnte. Auf diese Weise konnten Beschreibungen ausgewählter Ereignistypen im Videofilm sowie Beschreibungen zur Texteingabe und Textbearbeitung in einem Transkript an einer gemeinsamen Zeitachse dargestellt und bei der Analyse aufeinander bezogen werden.

Jedes Transkriptdokument im Korpus beschreibt die ChatTeilnahme eines Probanden. Der Fokus der Beschreibung liegt auf einer genauen Darstellung der Prozessualität der Beitragsproduktion sowie (für ein Kernkorpus von sechs Transkripten) auf einer Dokumentation, wann die visuelle Aufmerksamkeit der Probanden auf: welche Bereiche des Computerarbeitsplatzes fokussiert war. Die Beschreibung der Blickrichtung lässt sich über die gemeinsame Zeitachse wiederum auf die Eintreffenszeitpunkte neuer Beiträge im Bildschirmverlaufsprotokoll beziehen. Dadurch werden Annahmen darüber möglich, wann ein Proband einen am Bildschirm angezeigten Partnerbeitrag frühestens wahrgenommen haben kann und inwiefern die Kenntnisnahme des Partnerbeitrags ggf. zu Veränderungen bei der Beitragsproduktion geführt hat.

Das Setting, das der Datenerhebung zugrunde lag, war das einer kostenlosen, per Chat durchgeführten und nicht-moderierten Beratung zum Thema „eBay und Online-Auktionen“ durch eine aus Presse und 
TV bekannte Expertin. Die insgesamt 18 Chats wurden in verschiedenen Konstellationen durchgeführt, bei denen jeweils ein bis vier Probanden gleichzeitig mit der Expertin kommunizierten. Insgesamt wurden dabei 25 Std. 13 Minuten ,screen movie“" und 28 Std. 43 Minuten Videofilm erhoben. Die Chats dauerten insgesamt 11 Std. 26 Minuten, je Chat wurde das Chat-Verhalten mehrerer Probanden beobachtet. Die Probanden befanden sich während der Durchführung der Chats in unterschiedlichen Räumen und kannten sich vor ihrer Teilnahme am Experiment i.d.R. nicht. Bei der Probandenauswahl wurden sowohl Probanden mit geringer als auch Probanden mit großer Chat-Vorerfahrung berücksichtigt. Das Datenerhebungsdesign ist ausführlich in Beißwenger (2007: 287-336) beschrieben. ${ }^{1}$

Für 17 der 32 Probanden liegen im Korpus Transkripte vor, die die zeitliche Entwicklung des Verlaufsprotokolls und die Beitragsproduktion beschreiben. In sechs Transkripten ist darüber hinaus das Blickrichtungsverhalten komplett transkribiert (Kernkorpus). ${ }^{12}$ Die 17 Probanden verschicken in den etwa 11,5 Stunden ihrer Chat-Teilnahme insgesamt 889 Beiträge. Auf diese 889 Beiträge kommen insgesamt 635 textuell manifeste Revisionen. Minimale Revisionen, die nur ein Zeichen umfassen, wurden hierbei nicht oder nur in Ausnahmefällen mitgezählt. Der Umfang der Revisionen ist fallweise sehr unterschiedlich und umfasst zwischen 2 und 185 Tastaturanschlägen. Die Revisionen können sich auf den kompletten eingegebenen Text oder auch nur auf Textteile beziehen. 55\% aller Revisionen werden direkt, d.h. unmittelbar nach Eingabe des Bezugsausdrucks, ausgeführt, $45 \%$ nachträglich, d.h. nicht an dem Ausdruck, dessen rechter Rand zugleich den rechten Rand der aktuellen Texteingabe bildet, sondern an einer im linearen Textverlauf zurückliegenden Position.

Mit Blick auf den Produktionsdruck beim Chatten sowie auf die Dialogizität des Austauschs und die synchrone Kopräsenz der Partner

11 Chat-Kommunikation auf Basis von Daten aus Nutzerbeobachtungen zu erforschen, ist nicht neu, hat aber bislang - sicherlich auch aufgrund des im Vergleich zu rein mitschnittsbasierter Analyse hohen Aufwands bei der Datenerhebung und -aufbereitung nur wenig Verbreitung gefunden. Mit Daten, die den von mir erhobenen ,screen movies" vergleichbar sind, arbeiteten (in Forschungszusammenhängen mit z.T. unterschiedlichen Fragestellungen) bereits Garcia/Jacobs (1998; 1999); Vronay/Smith/Drucker (1999); Jones (2001), Ogura/Nishimoto (2004) sowie Markman (2006). Neu in dem von mir konzipierten Forschungsdesign ist die Miteinbeziehung von Videodaten zum Blickrichtungsverhalten. Eine neuere Arbeit, die ebenfalls „screen movies“ und Videobeobachtung integriert, ist Marcoccia/Atifi/Gauducheau (2008).

12 Vier Transkripte aus dem Kernkorpus können online unter http://www.michaelbeisswenger.de/sprachhandlungskoordination/ eingesehen werden. 
werde ich im Folgenden unterscheiden zwischen Revisionen, die durch die Wahmehmung von Partnerbeiträgen während der Texteingabe ausgelöst werden, und solchen Revisionen, die ohne Verarbeitung neu angezeigter Kommunikationsbeiträge auftreten. In Abschnitt 3.1 fasse ich die Ergebnisse einer in Beißwenger (2007) durchgeführten Fallstudie zusammen, Abschnitt 3.2 bringt eine explorative Klassifikation von intrinsisch motivierten Revisionen, die für den vorliegenden Beitrag neu aus den erhobenen Daten entwickelt wurde.

\subsection{Durch Partnerbeiträge (,extrinsisch“) motivierte Revisionen}

Revisionen, die auf eine zwischenzeitliche Verarbeitung von Partnerbeiträgen zurückgeführt werden können, nenne ich extrinsisch motiviert. Die Revision des aktuell in Verbalisierung befindlichen Handlungsplans wäre unter monologischen Bedingungen der Beitragsproduktion nicht aufgetreten. Der Anlass zur Revision kommt von außen, allerdings nicht in dem Sinne, dass der Partner direkt und willentlich auf die Beitragsproduktion einwirkt (die für ihn nicht transparent ist), sondern dahingehend, dass der Produzierende einen oder mehrere Partnerbeiträge nach dem Zeitpunkt ihrer Entdeckung in seine weitere Planung miteinbezieht.

Bedingt durch das en bloc-Prinzip werden Chat-Beiträge erst nach Abschluss des Produktionsprozesses für die Partner wahmehmbar. Die Möglichkeit zur Abstimmung der eigenen Handlungsplanung auf das Handeln der Partner besteht somit immer erst im Nachhinein zur bereits abgeschlossenen Verbalisierung. Selbst dann, wenn der Adressat im Augenblick der Anzeige eines neuen Beitrags seine visuelle Aufmerksamkeit auf das Bildschirmverlaufsprotokoll gerichtet hat und den Beitrag unmittelbar verarbeitet, weicht der Zeitpunkt, zu welchem die Handlungsentscheidung, die dem Beitrag unterliegt, für den Produzenten emergent wurde, um diejenige Zeitspanne vom Verarbeitungszeitpunkt ab, die es den Produzenten kostete, den Beitrag zu enkodieren und $\mathrm{zu}$ verschicken. Wenn der Produzent nach Verschickung seines Beitrags nicht in den Rezeptions- oder Wartemodus gewechselt hat, kann es sein, dass zum gleichen Zeitpunkt, zu welchem das Produkt seiner Versprachlichung sein Handeln für den Adressaten aktuell werden lässt, er selbst bereits mit der Planung und sprachlichen Umsetzung neuer Handlungspläne befasst ist. Dass zwei Kommunikationsbeteiligte zum selben Zeitpunkt einen exakt identischen Stand kognizieren, ist somit eher unwahrscheinlich (da zu dem, was mental als aktueller Stand vermerkt wird, neben rezipierten Partnerbeiträgen auch Entscheidungen 
über das individuelle Folgehandeln bzw. Handlungspläne gehören, die aktuell erst versprachlicht werden und den Partnern daher noch nicht zugänglich sind).

Aufgrund der visuellen Qualität des schriftlichen Mediums kann bei der Produktion eines eigenen Beitrags die Entwicklung des Kommunikationsverlaufs, so wie er sich am Bildschirm darbietet, weiterhin immer nur punktuell verfolgt werden: Sowohl die Produktionsaktivität (Tippen auf der Tastatur, Kontrolle des eingegebenen Textes) als auch die Überwachung des Verlaufsprotokolls erfordern visuelle Aufmerksamkeit (vgl. Abschnitt 2). Die Planung des eigenen Folgehandelns geht immer von demjenigen Stand aus, der - auf Basis der bereits rezipierten Partnerbeiträge - im mentalen Kommunikationsprotokoll eines Kommunizierenden vermerkt ist. ${ }^{13}$ Im Gegensatz zu mündlichen Gesprächen, bei denen aufgrund der materialen Qualität des phonischen Mediums die wechselseitige Wahrnehmung von Verhaltensäußerungen vollständig synchronisiert ist, steigt mit jeder Sekunde, die ein ChatBeteiligter nicht (mehr) auf das Verlaufsprotokoll am Bildschirm blickt, die Chance, dass das mentale Kommunikationsprotokoll vom Stand des Bildschirmverlaufsprotokolls abweicht, indem neue Partnerbeiträge (die beim letzten Blick des Beteiligten auf: das Bildschirmprotokoll noch nicht angezeigt waren) hinzukommen. Es ist ein Typikum der Kommunikationsteilhabe beim Chatten, dass $\mathrm{zu}$ einem bestimmten Zeitpunkt ein Teilnehmer auf Basis seines mental vermerkten Standes eine Handlungsentscheidung getroffen hat und diese versprachlicht, während das Bildschirmverlaufsprotokoll bereits einen Stand repräsentiert, nach welchem die in Versprachlichung befindliche Handlung gar nicht mehr erforderlich wäre. Überprüft der Produzierende bis zum Abschluss seines Produktionsprozesses und zur Verschickung seines fertigen Beitrags nicht erneut das Bildschirmverlaufsprotokoll, kommt es im Protokoll zu Dopplungen oder vermeintlich inkohärenten Sequenzen, die sich aus der temporären Divergenz des individuellen Kommunikationsprotokolls (des betreffenden Chatters) vom Stand des Bildschirmverlaufs erklären. Am Bildschirm muten die resultierenden

13 Der Begriff des mentalen Kommunikationsprotokolls wird hier in Anlehnung an Herrmann/Grabowski (1994: 336f.) für die mentale Repräsentation der Diskursstruktur verwendet, die von jedem Kommunizierenden auf Basis verarbeiteter Wahmehmung kommunikationsbegleitend mitgefiihrt wird. Das Kommunikationsprotokoll stellt den jeweils aktuellen Stand des Kommunikationsverlaufs bereit, auf dessen Basis u.a. Entscheidungen zur Produktion eigener Äußerungen getroffien werden. Zur Adaption des Konzepts des mentalen Kommunikationsprotokolls für die Modellierung der Kommunikationsteilhabe beim Chatten vgl. Beißwenger (2007: 163-171). 
Beitragssequenzen als thematische oder handlungssequenzielle Brüche bzw. als Redundanzen an; entsprechend können Erklärungen, die ausschließlich auf: Basis von Mitschnittsdaten getroffen werden, zu Fehldeutungen führen.

Überprüft der Produzierende während seines Produktionsprozesses erneut das Bildschirmverlaufsprotokoll, stellt sich ihm die Notwendigkeit, sein mentales Kommunikationsprotokoll ,upzudaten“ und den aktuell verfolgten Handlungsplan gegen den veränderten Stand zu evaluieren. Stellt er dabei fest, dass sein aktuell in Enkodierung befindliches Beitragsvorhaben nicht mehr ,passt“ (d.h. nicht mehr ohne Weiteres kohärent an den Stand des Bildschirmverlaufs anschließbar oder redundant geworden ist), hat er drei Möglichkeiten:

a) die Versprachlichung seines Beitragsplans ersatzlos aufzugeben;

b) trotz der entgegen der ursprünglichen Bedingungen für ungünstiger befundenen Ausgangsbedingungen für die Veräußerung seines anvisierten Beitrags diesen Beitrag dennoch zu Ende zu produzieren und abzuschicken;

c) auf die Fertigstellung seines anvisierten Beitrags zu verzichten, um stattdessen die Versprachlichung eines alternativen, unter den gegenwärtigen Bedingungen als zielführender oder relevanter bewerteten Handlungsplans in Angriff zu nehmen.

Im Rahmen einer Fallstudie, die in Beißwenger (2007: 367-465) ausführlich beschrieben ist, konnte gezeigt werden, dass von 1.097 Fällen, in denen Chatter mit der Texteingabe beginnen, 208 Fälle $(=19 \%)$ mit einer vollständigen Löschung des eingegebenen Textentwurfs abgeschlossen werden. Für eine Auswahl dieser Fälle wurde eine qualitative Analyse durchgeführt, die unter Einbeziehung der Daten zum Blickrichtungsverhalten sowie des kommunikativen Kontexts zu klären versuchte, wie viele Fälle von Komplettlöschung mit hoher Wahrscheinlichkeit durch die zwischenzeitliche Verarbeitung neuer Partnerbeiträge motiviert sind. Im Ergebnis sind $71 \%$ aller Komplettlöschungen auf die Verarbeitung von Partnerbeiträgen zurückzuführen, was nahelegt, dass Chatter bevorzugt die o.a. Varianten a. und c. wählen, wenn während der Beitragsproduktion neue Partnerbeiträge verarbeitet werden. Eine kohärente Anschließbarkeit eigener Beiträge ist im Chat also - ebenso wie im mündlichen Gespräch - höchst wichtig und in vielen kritischen Fällen aktivitätsleitendes Prinzip. Dass in der Chat-Kommunikation, auch wenn die Verlaufsprotokolle (oder Mitschnitte davon) bisweilen unübersichtlich anmuten, Kohärenz eine weniger wichtige Rolle spielen 
würde als im Gespräch, lässt sich nach diesen Befunden nicht bestätigen.

Gründe, die im Zuge der Kontextanalyse für die Löschung eingegebener Beitragsentwürfe identifiziert werden konnten, sind u.a.:

- Der Chatter hat mit seinem Entwurf eine Ergänzungsfrage (teil-) versprachlicht, die auf die Gewinnung einer Information zielt, die mit einem zwischenzeitlich eingetroffenen Partnerbeitrag bereits unabhängig bereitgestellt wurde; der aktuell in Versprachlichung befindliche Handlungsplan hat sich somit erledigt;

- der Chatter wird mit einem zwischenzeitlich eingetroffenen Partnerbeitrag von dessen Produzenten direkt adressiert (in der Form „@<username>“) und/oder ihm wird mit einem zwischenzeitlich eingetroffenen Partnerbeitrag eine Frage gestellt; der aktuell in Versprachlichung befindliche Handlungsplan wird aufgegeben, um direkt auf den neuen Partnerbeitrag mit Adressierung zu replizieren bzw. um unmittelbar der mit der Frage etablierten Folgehandlungsobligation nachzukommen;

- ein zwischenzeitlich eingetroffener Partnerbeitrag stellt eine Antwort auf eine zuvor vom Produzenten geäußerte Frage oder eine Anknüpfung an ein zuvor vom Produzenten initiiertes Thema dar. ${ }^{14}$

Ein Beispiel für eine extrinsisch motivierte Komplettlöschung ist in der Transkriptfläche in Beispiel (4) wiedergegeben. Da für die Bewertung der Revision als extrinsisch motiviert neben den individuellen Texteingabe- und -bearbeitungsaktivitäten auch die Zeitlichkeit der Produktion, die Zeitpunkte der Veränderung des Bildschirmverlaufsprotokolls sowie das im betreffenden Zeitraum beobachtbare Blickrichtungsverhalten der Chatterin dargestellt werden müssen, kann in diesem Fall auf ein Transkript, welches die verschiedenen Datentypen zueinander in Beziehung setzt, nicht verzichtet werden. ${ }^{15}$

14 Für weitere mögliche Gründe vgl. Beißwenger (2007: 461ff.) sowie die zugehörigen Einzelfallanalysen.

15 Das Transkript ist wie folgt aufgebaut: Die Spalte „ZEIT“ gibt - im Format < Stunde:Minuten:Sekunden> - die Realzeitpunkte an, denen die in den übrigen Spalten beschriebenen Ereignisse und Aktivitäten zuzuordnen sind. Die Spalte „Protokollverlauf“ vermerkt jeweils sekundengenau das Eintreffen neuer Beiträge im Bildschirmverlaufsprotokoll und gibt sie im Wortlaut der Anzeige wieder. Die Spalte „Produktionsaktivitäten“ gibt für den Chattenden, dessen Kommunikationsteilhabeaktivitäten beobachtet wurden, die am Bildschirm beobachtbaren Produktionsaktivitäten wieder (Texteingabe und -bearbeitung). Text, der innerhalb derselben Tabellenzelle steht, wurde vom betreffenden Chatter jeweils kontinuierlich, d.h. mit nicht mehr als 2.0 Sekunden Unterbre- 
Das Transkript beschreibt die Kommunikationsteilhabe der Chatterin INAB. Neben ihr und der Expertin (BSOMMER) ist am Chat noch eine weitere Chatterin (FALA) beteiligt. Der Beitrag von BSOMMER, der seit 10:44:59 im Verlaufsprotokoll angezeigt wird, ist INAB bekannt. Da INAB bei der Rezeption von Beiträgen diese häufig tonlos mit den Lippen mitartikuliert, liefert ihre im Video dokumentierte Gesichtspartie neben dem Blickrichtungsverhalten zusätzliche Anhaltspunkte dafür, wann sie Partnerbeiträge rezipiert.

Um 10:45:09 beginnt INAB mit der Enkodierung eines eigenen Beitrags, die sich nonverbal bereits um 10:45:08 mit einem Wechsel des Hauptblickfokus vom Verlaufssprotokoll auf die Tastatur ankündigt. Um 10:45:12, spätestens 10:45:14, bemerkt INAB im Verlaufsprotokoll einen neu angezeigten Beitrag von BSOMMER, der als Nachtrag zum Beitrag aus 10:44:59 gelten kann. INABs Blick fokussiert daraufhin für drei Sekunden auf das Texteingabeformularfeld; vermutlich evaluiert sie, ob der Plan, der mit dem eingegebenen Segment ich denke bereits teilweise realisiert wurde, noch zielführend ist. Die Löschung um 10:45:18 legt nahe, dass negativ evaluiert wurde. Der Löschung folgt unmittelbar eine erneute Eingabe von Text (10:45:20). INAB verwirft also nicht nur ihren zuvor verfolgten Plan, sondern hat bereits einen alternativen oder modifizierten Plan parat, der nun alternativ versprachlicht werden soll. Auch dieser Plan wird durch das Eintreffen eines neuen Partnerbeitrags (FALA um 10:45:21, bemerkt um 10:45:22) aufgegeben (Löschung ab

chung, in das Texteingabeformularfeld der Bildschirmoberfläche eingegeben. Durchgestrichener Text beschreibt jeweils einen Löschvorgang, die über dem betreffenden Textsegment ausgefiihrt wurde. Hochgestellte Ziffern sind Referenzziffern, die dazu dienen, in nachgeordneten Teilen des Transkripts auf die betreffenden Stellen im linearen Textverlauf Bezug zu nehmen, um nachträglich an einer im Textverlauf zurückliegenden Position ausgeführte Revisionen zu beschreiben. Solche nachträglichen Revisionen sind durch Wiederholung der entsprechenden Referenzziffer gekennzeichnet. Die Spalte „Blick“ beschreibt das zentrale Blickrichtungsziel des beobachteten Chatters für einzelne Zeiträume seiner Chat-Teilnahme: Im Falle der Angabe „Pro“ liegt der Hauptblickfokus auf dem Bereich der Benutzeroberfläche, in welchem das Bildschirmverlaufsprotokoll angezeigt wird; ist der Angabe „Pro“ das Symbol $\left.<^{\circ}-^{\circ}-^{\circ}\right\rangle$ zugefügt, indiziert das, dass sich im Videofilm zusätzlich eindeutige Pupillenbewegungen in Textverlaufsrichtung feststellen ließen. Im Falle der Angabe „Key“ liegt die Hauptblickrichtung auf der Tastatur (Key board), bei „Form“ liegt der Blickfokus auf dem Formularfeld für die Eingabe eigener Beiträge (Texteingabeformularfeld) der Bildschirmoberfläche. „(off)“ gibt an, dass der Blick nach außerhalb des Computerarbeitsplatzes gerichtet ist. Die Spalte „Anmerkungen“ beschreibt in nicht-standardisierter Form Beobachtungen zu weiteren Verhaltensmodi - in Beispiel 4 etwa zur Körperpositur, zur Mimik und zur rezeptionsbegleitenden mündlichen Verbalisierung der Chatterin. - Eine ausführliche Beschreibung und Begründung des Transkriptionsformats findet sich in Beißwenger (2007: 336363). 
10:45:28). Anschließend bleibt INAB für mindestens 11 Sekunden in Rezeptionshaltung (Blick aufs Verlaufsprotokoll und Pupillenbewegungen in Textverlaufsrichtung), erst ab 10:45:40 (Blick aufs Eingabeformularfeld, anschließend Texteingabe) setzt sie erneut zur Produktion an.

Beispiel (4): Extrinsisch (= durch die Verarbeitung neuer Partnerbeiträge) motivierte Komplettlöschungen:

\begin{tabular}{|c|c|c|c|c|}
\hline Protokollverlauf & ZEIT & $\begin{array}{l}\text { Produktionsak- } \\
\text { tivitäten (Bild- } \\
\text { schirm) INAB }\end{array}$ & Blick & $\begin{array}{l}\text { Anmerkun- } \\
\text { gen }\end{array}$ \\
\hline & $10: 44: 50$ & & \multirow{3}{*}{$\begin{array}{l}\text { Pro } \\
\mathrm{O}^{-} \mathrm{O}^{-} \mathrm{O}\end{array}$} & \multirow{4}{*}{$\begin{array}{l}\text { stützt das } \\
\text { Kinn in die } \\
\text { Hand }\end{array}$} \\
\hline \multirow[t]{6}{*}{$\begin{array}{l}\text { bsommer: Zum Glück } \\
\text { sind die meisten eBayer } \\
\text { ehrliche Menschen, die } \\
\text { nichts Böses im Schilde } \\
\text { führen. }\end{array}$} & $10: 44: 59$ & & & \\
\hline & $10: 44: 05$ & & & \\
\hline & $10: 45: 06$ & & Form & \\
\hline & $10: 45: 07$ & & Pro & \multirow{6}{*}{$\begin{array}{l}\text { spricht hin } \\
\text { und wieder } \\
\text { einige } \\
\text { Wörter } \\
\text { lautlos mit }\end{array}$} \\
\hline & $10: 45: 08$ & & Key & \\
\hline & $10: 45: 09$ & \multirow[t]{3}{*}{ ich denke, } & & \\
\hline \multirow[t]{6}{*}{$\begin{array}{l}\text { bsommer: Zumindest } \\
\text { die Privatverkäufer ... }\end{array}$} & $10: 45: 11$ & & & \\
\hline & $10: 45: 12$ & & \multirow{2}{*}{$\begin{array}{l}\text { Pro | } \\
\text { Form | } \\
\text { Key }\end{array}$} & \\
\hline & $10: 45: 13$ & & & \\
\hline & $10: 45: 14$ & & Pro & \multirow[b]{2}{*}{$\begin{array}{l}\text { beugt sich } \\
\text { weiter zum } \\
\text { Bildschirm } \\
\text { vor; leicht } \\
\text { irritierter } \\
\text { Ge- } \\
\text { sichtsaus- } \\
\text { druck, } \\
\text { spricht } \\
\text { lautlos mit }\end{array}$} \\
\hline & $10: 45: 15$ & & \multirow[t]{2}{*}{ Form } & \\
\hline & $10: 45: 18$ & ieh-denke; & & \\
\hline
\end{tabular}




\begin{tabular}{|c|c|c|c|c|}
\hline Protokollverlauf & ZEIT & $\begin{array}{l}\text { Produktionsak- } \\
\text { tivitäten (Bild- } \\
\text { schirm) INAB }\end{array}$ & Blick & $\begin{array}{l}\text { Anmerkun- } \\
\text { gen }\end{array}$ \\
\hline & $10: 45: 19$ & & Pro & \\
\hline & $10: 45: 20$ & \multirow[t]{2}{*}{ kenn } & \multirow[t]{2}{*}{ Key } & \\
\hline \multirow[t]{9}{*}{$\begin{array}{l}\text { fala: aber meine ur- } \\
\text { sprüngliche frage ist } \\
\text { noch nicht ganz abge- } \\
\text { schlossen; ebay wird nie } \\
\text { von selbst aktiv, oder }\end{array}$} & $10: 45: 21$ & & & \\
\hline & $10: 45: 22$ & & \multirow{2}{*}{$\begin{array}{l}\text { Pro } \\
0^{-} 0^{-} \circ\end{array}$} & \multirow{2}{*}{$\begin{array}{l}\text { spricht } \\
\text { lautlos mit }\end{array}$} \\
\hline & $10: 45: 28$ & \multirow[t]{2}{*}{ kenn } & & \\
\hline & $10: 45: 29$ & & Form & \\
\hline & $10: 45: 30$ & & \multirow{2}{*}{$\begin{array}{l}\text { Pro } \\
0^{-} 0^{-} \circ\end{array}$} & \multirow{2}{*}{$\begin{array}{l}\text { stïtzt das } \\
\text { Kinn in die } \\
\text { Hand }\end{array}$} \\
\hline & $10: 45: 39$ & & & \\
\hline & $10: 45: 40$ & & Form & $\begin{array}{l}\text { legt die } \\
\text { Hände an } \\
\text { den Nacken }\end{array}$ \\
\hline & $10: 45: 41$ & \multirow{3}{*}{$\begin{array}{l}\text { das wär ja nen } \\
\text { Ding } \\
\text { VERSCHI- } \\
\text { CKEN }\end{array}$} & \multirow[t]{2}{*}{ Key } & \multirow{2}{*}{$\begin{array}{l}\text { spricht } \\
\text { lautlos } \\
\text { einige } \\
\text { Wörter mit }\end{array}$} \\
\hline & $10: 45: 46$ & & & \\
\hline $\begin{array}{l}\text { bsommer: Apropos } \\
\text { Privatverkäufer: Ich } \\
\text { gehe davon aus, dass Sie } \\
\text { vorhin das Rückgabe- } \\
\text { recht bei privaten Ver- } \\
\text { käufen meinten, oder? } \\
\text { inab: das wär ja nen } \\
\text { Ding }\end{array}$ & $10: 45: 47$ & & $\begin{array}{l}\text { Prol } \\
\text { Form }\end{array}$ & \\
\hline
\end{tabular}

Die Komplettlöschung ist nur eine, wenn auch vergleichsweise häufige, Form der extrinsisch motivierten Revision. Daneben kommen nachträgliche Hinzufügungen, Löschungen oder Ersetzungen von eingegebenen Textteilen vor, mit denen nicht der Handlungsplan als Ganzer revidiert, sondern die Gestaltung der Äußerung den aktuellen Gegebenheiten (dem veränderten Stand) angepasst wird. Der Transkriptausschnitt in Beispiel (5) beschreibt einen Fall nachträglicher Hinzufügung (gekennzeichnet mit der Sigle $\underline{\text { Add) }}$. Der Chatter MAGE ist, nach einer nicht 
extrinsisch motivierten Löschung um 13:27:03, ${ }^{16}$ ab 13:27:05 mit der Enkodierung eines Handlungsplans befasst, mit welchem er eine Frage realisieren möchte, die sich auf den Beitrag von BSOMMER bezieht, der seit 13:26:55 im Verlaufsprotokoll angezeigt wird. MAGEs Blickrichtungsfokus zwischen 13:26:56 und 13:27:01 legt nahe, dass ihm dieser Beitrag von BSOMMER bekannt ist und sein Folgehandeln daher darauf bezogen werden kann. Das deiktische Element da (genau da setzt eine meiner fragen an) stützt diese Annahme, da es unwahrscheinlich wäre, dass MAGE mittels $d a$ an einen Beitrag anschließt, der in seinem mentalen Kommunikationsprotokoll nicht den seiner eigenen Versprachlichung unmittelbar vorausgehenden Beitrag darstellt.

Während der Eingabe des Textsegments genau da setzt eine meiner fragen an: fällt MAGEs Blick (ab13:27:09) erneut auf das Verlaufsprotokoll, in dem inzwischen (um 13:27:08 und um 13:27:09) zwei neue Beiträge der Chat-Partner SASCHU und CHRE angezeigt werden. MAGE unterbricht seine Texteingabe; sein Blick liegt für weitere 5 Sekunden auf dem Verlaufsprotokoll. Nachdem er die beiden neuen Beiträge offensichtlich rezipiert hat, blickt er wieder auf die Tastatur (13:27:15), platziert den Cursor vor dem bereits eingegebenen Text und stellt diesem durch nachträgliche Hinzufügung (13:27:16-13:27:20) ein Adressierungselement (@bsommer:) voran,um die Beziehbarkeit seines Beitrags auf den - bislang in seinem mentalen Kommunikationsprotokoll als unmittelbarer Vorbeitrag geführten - Beitrag von BSOMMER auch weiterhin sicherzustellen. Während eine explizite Adressierung des Partners bis 13:27:09 nicht erforderlich erschien (BSOMMERs Beitrag wurde bis zu diesem Zeitpunkt als letzter geäußerter Beitrag in MAGEs mentalem Protokoll geführt, der Beitrag von SASCHU um 13:27:08 war ihm noch nicht bekannt), hat sich der Stand für MAGE insoweit verändert, als der Bezug seines Beitrags auf den Beitrag von BSOMMER nun nicht mehr per Default möglich ist (,,im Normalfall und falls nicht anders angezeigt, bezieht sich ein Beitrag auf den unmittelbar davor angezeigten Beitrag"), sondern aufgrund größerer Entfernung sprachlich explizit gemacht werden muss. 
Beispiel (5): Extrinsisch motivierte nachträgliche Hinzufügung eines Adressierungselements:

\begin{tabular}{|c|c|c|c|}
\hline Protokollverlauf & ZEIT & $\begin{array}{l}\text { Produktionsaktivitä- } \\
\text { ten (Bildschirm) } \\
\text { MAGE }\end{array}$ & Blick $^{17}$ \\
\hline \multirow[t]{7}{*}{$\begin{array}{l}\text { bsommer: Sie haben allerdings } \\
\text { auch bei privaten Verkäufen ein } \\
\text { Rückgaberecht, wenn die Ware } \\
\text { defekt ist oder nicht der Beschrei- } \\
\text { bung entspricht. }\end{array}$} & $13: 26: 55$ & & \\
\hline & $13: 26: 56$ & & \multirow[t]{2}{*}{ Pro } \\
\hline & $13: 27: 01$ & & \\
\hline & 13:27:02 & dabei & Key \\
\hline & 13:27:03 & \multirow[t]{2}{*}{ dabei } & Form \\
\hline & 13:27:04 & & \multirow[t]{3}{*}{ Key } \\
\hline & $13: 27: 05$ & \multirow{3}{*}{$\begin{array}{l}\text { 'genau da setzt eine } \\
\text { meiner fragen an: }\end{array}$} & \\
\hline $\begin{array}{l}\text { saschu: wenn der angebotene } \\
\text { Artikel stark von der Artikelbe- } \\
\text { schreibung abweicht kann man } \\
\text { auch bei Privat umtauschen }\end{array}$ & $13: 27: 08$ & & \\
\hline \multirow[t]{6}{*}{$\begin{array}{l}\text { ehre: Ich habe das so verstanden, } \\
\text { dass Ebay-Auktionen im Grunde } \\
\text { Fernhandelskäufe (oder so) sind } \\
\text { und somit ein ganz normales Ver- } \\
\text { tragsrücktrittsrecht besteht (bin } \\
\text { aber kein Jurist). }\end{array}$} & 13:27:09 & & \multirow[t]{2}{*}{ Pro } \\
\hline & 13:27:14 & & \\
\hline & $13: 27: 15$ & & \multirow[t]{4}{*}{ Key } \\
\hline & $13: 27: 16$ & \multirow[t]{2}{*}{$\underline{\text { Add }}^{1} @$ bsommer: } & \\
\hline & $13: 27: 20$ & & \\
\hline & $13: 27: 21$ & inwiewiet kann man & \\
\hline
\end{tabular}

17 Die Angaben zur Blickrichtung wurden gegenüber dem Originaltranskript vereinfacht. Angegeben ist jeweils lediglich die Hauptblickrichtung. Kleinere Blickabschweifungen auf andere Bereiche des Computerarbeitsplatzes sind nicht dargestellt. Das vollständige Transkript kann online unter http://www.michael-beisswenger.de/sprachhandlungskoordination/ eingesehen werden. 


\begin{tabular}{|c|c|c|c|}
\hline Protokollverlauf & ZEIT & $\begin{array}{l}\text { Produktionsaktivitä- } \\
\text { ten (Bildschirm) } \\
\text { MAGE }\end{array}$ & Blick $^{17}$ \\
\hline & $13: 27: 46$ & $\begin{array}{l}\text { den Angaben trauen. } \\
\text { ich hatte einen fall, } \\
\text { wo ich ein wetten- } \\
\text { dass geselsl- } \\
\text { chaftsspiel ersteigert } \\
\text { habe, für } 10 \text { euro }\end{array}$ & \\
\hline & $13: 27: 47$ & & (off) \\
\hline & $13: 27: 50$ & & \\
\hline & $13: 27: 51$ & & Key \\
\hline & $13: 27: 52$ & \multirow{4}{*}{$\begin{array}{l}\text {, im netz war eine } \\
\text { glanzvolle verpa- } \\
\text { ckung angegebe- } \\
\text { zeigt, dabei war das } \\
\text { spielt schon alt, aus } \\
\text { den } 80 \mathrm{ern} \text {, als aus- } \\
\text { rede kam: „Oh, das } \\
\text { war wohl ein Verse- } \\
\text { hen“. } \\
\text { VERSCHICKEN }\end{array}$} & \\
\hline $\begin{array}{l}\text { bsommer: Bei Spiegel Online } \\
\text { findet sich hierzu folgendes: }\end{array}$ & $13: 28: 01$ & & \\
\hline$[\ldots]$ & {$[\ldots]$} & & \\
\hline $\begin{array}{l}\text { mage: @bsommer: genau da setzt } \\
\text { eine meiner fragen an: inwiewiet } \\
\text { kann man den Angaben trauen. ich } \\
\text { hatte einen fall, wo ich ein wetten- } \\
\text { dass geselslchaftspiel ersteigert } \\
\text { habe, für } 10 \text { euro, im netz war eine } \\
\text { glanzvolle verpackung angezeigt, } \\
\text { dabei war das spielt schon alt, aus } \\
\text { den } 80 \text { ern, als ausrede kam: „Oh, } \\
\text { das war wohl ein Versehen“. }\end{array}$ & $13: 28: 25$ & & \\
\hline
\end{tabular}

\subsection{Nicht durch Partnerbeiträge (,intrinsisch“) motivierte Revisionen}

Neben den beschriebenen, der Kohärenz geschuldeten Revisionen gibt es eine große Gruppe von Revisionen, die als kleine monologische Textproduktionsprozesse aufgefasst werden können. Bei diesen wird durch eine punktuelle Evaluation der verschickten Produkte der zeitlich kopräsenten (aber ansonsten, auch in ihren Aktivitäten, unsichtbaren) Partner ein Schreibanlass eingeholt, weitere Beiträge der Partner spielen dann aber für die Beitragsproduktion bis auf weiteres keine Rolle. Bezogen auf den Stand des Kommunikationsverlaufs, der sich nach einer Evaluation des Bildschirmverlaufsprotokolls jeweils darbietet und der im individuell geführten mentalen Protokoll als aktueller Kontext 
eingetragen wird, wird die eigene Handlungsplanung so adaptiert, dass sie zum aktuellen Stand passt. Resultiert aus einer solchen Anpassung der individuellen Agenda der Entschluss, selbst produktiv tätig zu werden, um dem kognizierten Stand einen eigenen Handlungszug folgen zu lassen, so wird ein Verbalisierungsprozess in Gang gesetzt, der darauf zielt, den gefassten Handlungsplan sprachlich so umzusetzen, dass ein möglichst konstruktiver wie kohärenter Anschluss an den kognizierten Stand möglich ist. Ob und wie stark beim Beginn und während des Verbalisierungsprozesses der kognizierte Stand (d.h. derjenige Stand, der im mentalen Protokoll als aktueller Stand geführt wird) vom tatsächlichen, am Bildschirm dokumentierten Stand abweicht, spielt für die Verbalisierung (d.h. das Formulieren und schriftliche Enkodieren des Handlungsplans) so lange keine Rolle, als der Produzierende keine erneute Evaluation des Bildschirmverlaufsprotokolls vornimmt. Solange seine visuelle Aufmerksamkeit ausschließlich auf das Monitoring der eigenen Tippaktivitäten auf der Tastatur und des eingegebenen Textentwurfs im Eingabeformularfeld der Benutzeroberfläche verwendet wird, bleiben ggf. zwischenzeitlich hinzugekommene weitere Partnerbeiträge im Bildschirmverlaufsprotokoll unbemerkt und bleibt der kognizierte Stand des Kommunikationsverlaufs unverändert. Auftretende Textrevisionen können hier nicht als Anpassungen an zwischenzeitlich veränderte Kontextbedingungen erklärt werden, sondern als Ergebnisse von Formulierungsarbeit und Evaluationen des bereits enkodierten Textentwurfs. Sie können in dieser Hinsicht und gegenüber den unter 3.1 behandelten Revisionen als intrinsisch motiviert beschrieben werden.

Prototypische Fälle intrinsisch motivierter Revision sind direkt, d.h. unmittelbar nach Eingabe des Bezugsausdrucks, ausgeführte Ersetzungen, bei denen ein eingegebener Ausdruck, dessen rechtes Ende mit dem Ende der aktuell eingegebenen Zeichensequenz zusammenfällt, unmittelbar wieder gelöscht und durch einen alternativen Ausdruck ersetzt wird.

Für den vorliegenden Beitrag habe ich mein Kernkorpus, das etwa vier Stunden Chat-Teilnahme von insgesamt sechs Probanden dokumentiert, nach Revisionen durchsucht, die die folgenden Kriterien erfüllen:

- Der Löschvorgang wird nicht länger als 2.0 Sekunden nach Unterbrechung der Texteingabe ausgeführt;

- Gegenstand der Revision ist eine Folge von mindestens zwei Zeichen oder ein einzelnes eingegebenes Zeichen macht unter Einbe- 
ziehung des Kontexts eine anvisierte Wortform/eine anvisierte Fortsetzung von mindestens Wortlänge vermutbar;

- auf den Abschluss des Löschvorgangs folgt spätestens nach 2.0 Sekunden entweder erneute Texteingabe oder die Ausführung einer Verschickungshandlung;

- im Fall, dass der Produzierende während der Texteingabe, die der Löschung vorausgeht, auf das Bildschirmverlaufsprotokoll geblickt hat, gilt die Revision nur dann als intrinsisch motiviert, wenn zwischen diesem und dem letzten vor Beginn der Produktionsaktivität liegenden Blick aufs Protokoll kein neuer Partnerbeitrag ins Protokoll eingespielt wurde.

\begin{tabular}{|c|c|c|c|c|c|c|c|c|c|c|c|c|}
\hline & \multirow[b]{2}{*}{ 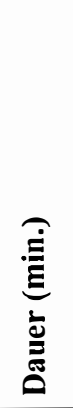 } & \multirow[b]{2}{*}{ 萬 } & \multicolumn{10}{|c|}{$\begin{array}{l}\text { intrinsische Direkt-Revisionen } \\
\text { (gesamt und nach Typen) }\end{array}$} \\
\hline & & & 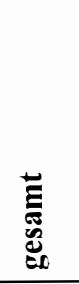 & 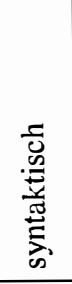 & 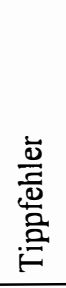 & 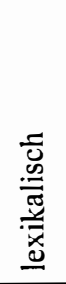 & 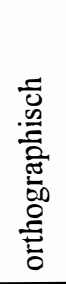 & 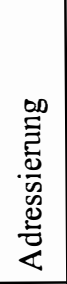 & 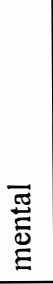 & 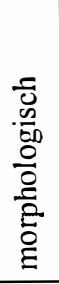 & 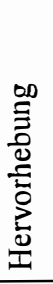 & 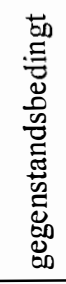 \\
\hline bensch & 37 & 77 & 68 & 29 & 26 & 7 & 5 & 3 & - & 2 & 1 & - \\
\hline larbe & 33 & 102 & 27 & 13 & 7 & 2 & 1 & - & 3 & - & - & 2 \\
\hline inab & 42 & 54 & 12 & 4 & 6 & 3 & - & - & - & - & - & - \\
\hline mage & 42 & 26 & 10 & 6 & 2 & 4 & - & - & - & - & - & - \\
\hline fala & 42 & 50 & 3 & 3 & - & - & - & - & - & - & - & - \\
\hline \multirow[t]{2}{*}{ jecom } & 41 & 38 & 2 & 2 & 1 & 1 & - & - & - & - & - & - \\
\hline & 237 & 347 & 122 & 57 & 42 & 17 & 6 & 3 & 3 & 2 & 1 & 2 \\
\hline
\end{tabular}

Abbildung 1: Übersicht: Direkt ausgeführte intrinsisch motivierte Revisionen im untersuchten Korpus. ${ }^{18}$

18 Die für die einzelnen Typen intrinsisch motivierter Revisionen ermittelten Häufigkeiten ergeben in der Summe mehr als die Gesamtzahl der vorgefundenen Revisionen. Das hat damit zu tun, dass in einigen Einzelfällen die Daten zwei verschiedene Deutungsmöglichkeiten zuließen. Solche Fälle wurden, wenn sich auch unter Einbeziehung des Kontexts keine eindeutige Entscheidung treffien ließ, doppelt klassifiziert und schlagen in 
Die Tabelle in Abbildung 1 gibt eine Übersicht über die Fälle intrinsisch motivierter Revisionen, die unter Anwendung der o.a. Kriterien im Kernkorpus identifiziert werden konnten. Insgesamt fanden sich 122 Belege, die allerdings recht unterschiedlich auf die einzelnen Chatter verteilt sind. Die erste Tabellenspalte listet die Namen der sechs Chatter, die Spalten 2 und 3 umfassen allgemeine Angaben zur Dauer der Chats sowie zur Anzahl der im Verlaufsprotokoll auf den Teilnehmerbildschirmen angezeigten (verschickten) Beiträge, die dem betreffenden Chatter als Urheber zugeordnet sind. Das Tabellensegment mit der Überschrift ,,intrinsische Direkt-Revisionen“ beziffert die Anzahl der Revisionen und ihre Verteilung auf eine Reihe von Revisionstypen, zu denen die Belege bei der Analyse gruppiert werden konnten. Sie können als ein Vorschlag zur Klassifikation von Revisionen in der ChatKommunikation verstanden werden. Die einzelnen Typen werden im Folgenden anhand einer Analyse ausgewählter Belegbeispiele erläutert.

\subsubsection{Syntaktische Revisionen}

Als syntaktisch lassen sich solche Revisionen beschreiben, bei denen der revidierte und der ersetzende Ausdruck unterschiedliche syntaktische Strukturen aufweisen oder bei denen der revidierte Ausdruck nicht mit derjenigen Struktur hätte fortgeführt werden können, mit der der ersetzende Ausdruck fortgeführt wird.

Syntaktische Revisionen sind Ausdruck von Umformulierungen auf verschiedenen sprachlichen Ebenen. Die Bandbreite reicht von Umformulierungen zum Zweck der semantischen Präzisierung über thematisch bedingte Umstellungen bis hin zu Umplanungen, bei denen entweder dieselbe Äußerung (oder Teile daraus) in einem alternativen als dem ursprünglich gewählten syntaktischen Muster realisiert oder die komplette Äußerung (oder Teile davon) syntaktisch und auch propositional umgebaut werden.

Die syntaktischen Revisionen stellen im untersuchten Korpus die größte Gruppe unter den intrinsisch motivierten Revisionen. Die folgenden Formen lassen sich unterscheiden:

der Tabelle daher doppelt zu Buche. Bei der Ermittlung der Gesamtzahl wurde hingegen jeder Beleg nur einmal gezählt. 
a. Präzisierung durch Ausbau:

Ein gerade verbalisierter oder in Verbalisierung befindlicher Ausdruck wird unmittelbar nach seiner Enkodierung oder während seiner Enkodierung umformuliert. Die Umformulierung führt $\mathrm{zu}$ einer Löschung des ganz oder teilweise enkodiert vorliegenden Ausdrucks und seiner Ersetzung durch einen Ausdruck, der gegenüber dem gelöschten Ausdruck ein zusätzliches charakterisierendes Element - z.B. ein zusätzliches Adverbial, eine koordinativ angeschlossene weitere Phrase in gleicher syntaktischer Position - beinhaltet. An der (geplanten) syntaktischen Linearstruktur wird dabei nichts geändert: ${ }^{19}$

19 Das Format für die Darstellung der Belege ist wie folgt aufgebaut: Grundsätzlich sind alle Aktivitäten, die während der Produktion im Eingabeformularfeld textuell manifest werden oder an bereits textuell manifesten Einheiten ausgeführt werden, in linearem Nacheinander dargestellt, auch wenn sie sich auf im Textverlauf bereits zurückliegende Einheiten beziehen. Die räumliche Abfolge von links nach rechts symbolisiert also primär die zeitliche Abfolge der Texteingabe- und -bearbeitungsaktivitäten und nur sekundär den Textverlauf. Die äußere geschweifte Klammerung kennzeichnet dasjenige Transkriptsegment, das die Revision repräsentiert; die innere geschweifte Klammerung kennzeichnet die Löschaktivität, mit welcher ein bestimmtes Textsegment aus dem Eingabeformularfeld gelöscht wird, um es anschließend durch einen alternativen Ausdruck zu ersetzen. Löschaktivität und -gegenstand sind durch eine mit Durchstreichung markierte Wiedergabe des gelöschten Ausdrucks beschrieben. Dasjenige Transkriptsegment, das zwischen der Beschreibung der Löschaktivität und der öffnenden äußeren Schweifklammer steht, beschreibt die Eingabe des später gelöschten Ausdrucks. Dasjenige Transkriptsegment, das zwischen der Beschreibung der Löschaktivität und der schließenden äußeren Schweifklammer steht, beschreibt denjenigen Ausdruck, der nach erfolgter Löschung an der Stelle des gelöschten Ausdrucks eingegeben wird und diesen ersetzt. In Fällen, in denen eine eindeutige Grenze des ersetzenden Ausdrucks zu daran anschließenden weiteren Texteingaben zu ziehen war, steht die schließende äußere Schweifklammer an der entsprechenden Stelle im Belegexzerpt; in Fällen, in denen die komplette nachfolgende Texteingabe als ersetzender Ausdruck anzusehen ist (z.B. Fälle von Umplanung, vgl. c.), fällt die Schweifklammer mit dem Ende des Belegexzerpts zusammen. Das Zeichen <.」> symbolisiert die Ausführung einer Verschickungshandlung, mit welcher der aktuelle Stand eines Textentwurfs zur Übermittlung an den Server übergeben wird. Auslassungspunkte in eckigen Klammem markieren, dass an der betreffenden Stelle des Belegexzerpts Teile des Produktionsprozesses nicht wiedergegeben wurden (i.d.R. deshalb, weil sie für das, was der Beleg belegen soll, nicht relevant sind). In eckige Klammern gesetzter Text ist Text, der (ggf: mit dazwischen liegenden Pausen oder anderen Aktivitäten) vor oder nach dem Belegexzerpt eingegeben wurde, aber offensichtlich der Realisierung desselben Äußerungsplans zuzurechnen ist; solche Textteile sind dann mit angegeben, wenn sie für das Verständnis des mit dem Belegexzerpt Versprachlichten erforderlich sind. Jedem Beleg ist eine Sigle nachgestellt, die sich aus dem Teilnehmernamen des Chatters zusammensetzt, dessen Textproduktionsaktivität aufgezeichnet wurde, sowie aus einer Angabe zum Zeitpunkt (Stunde:Minute:Sekunde) des Beginns der Produktionsaktivitäten, die im Exzerpt dokumentiert sind. 
(6) das man \{nicht unterscheiden \{nicht-unterseheiden manchmal nicht unterscheiden\} kann ob etwas privat oder komerziell ist und ich mich frage, ob kommerzielle anbieter bei ebay nicht von grunfd aufi(MAGE 13:22:50)

(7) eine schlechte bewertung abgeben, wenn der mich zuvor \{rei \{rei\} irgendwann mal rei\}ngelegt hat (INAB 10:42:01)

(8) Jeder Anbieter, der soetwas \{im Inte \{nte\}oder soetwas aehliches im Inte) rnet benutzt, wird verklagt. (LARBE 16:26:42)

(9) naja, in der Wut spricht man dinge, die \{an Übertre \{an-̈̈berfe\} schnell in Übertre\}ibung umschwenken. (MAGE 13:35:42)

Der umgekehrte Fall, bei welchem Adverbiale oder Attribute nachträglich getilgt werden, tritt ebenfalls in den Daten auf:

(10)CDs gibt's da manchmal \{ganz günstog \{ganz-günstøg\} günstig\}. (BENSCH 14:05:33)

(11)dass ich wenn ich $\{z . B$. etwas suche $\{z$ :B-etwas-suche $\}$ etwas suche $\}$, [seitenweise die gleichen Artikel angezeigt bekomme.] (BENSCH 13:58:31)

\section{b. Präzisierung durch Ersetzung:}

Ein gerade verbalisierter oder in Verbalisierung befindlicher Ausdruck wird unmittelbar nach seiner Enkodierung oder während seiner Enkodierung umformuliert. Die Umformulierung führt zu einer Löschung des ganz oder teilweise enkodiert vorliegenden Ausdrucks und seiner Ersetzung durch einen Ausdruck, bei dem ein Element des revidierten Ausdrucks durch ein Element mit gleicher syntaktischer Funktion (z.B. ein Adverbial) ersetzt ist, mit dem spezifischer charakterisiert werden kann:

(12) \{Irgendwann habe ich d \{frgendwann habe ieh d\}Nach mehreren Monaten habe ich n \}och mal eine Kopie meines Kontoauszuges an das Inkassounternehmen geschickt. (LARBE 16:17:09)

\section{c. Umplanung:}

Der bereits eingegebene Textentwurf wird ganz oder zurückgehend bis zum Startpunkt einer größeren syntaktischen Konstruktion (z.B. Satzoder Teilsatzgrenze) gelöscht und durch einen Ausdruck ersetzt, der gegenüber dem gelöschten Ausdruck einen alternativen Plan erkennen lässt:

(13)[woher weiß] ich, dass der anbeiter eines Produktes volljährig ist? \{Ich wi \{tw das kontrolliert doch keiner nach.\} (MAGE 13:44:35) 
(14)[Aber in letzter Zeit] sind sämtliche Kategorien dermaßen von den sog. Powersellern bevölkert, die \{mit\{mit\}hunderte gleiche Artikel anbieten\} (BENSCH 13:45:52)

(15) \{wenn \{went\} habe mal ne digitalkmaera gekauft die aber selbst abgeholt (FALA $11: 07: 35$ )

(16) $\{$ Ich ha\{ $\{$ Leider nicht mehr $\}$ (LARBE 16:29:01)

Die Umplanung kann dabei mehr oder weniger radikal sein. Während in Beispiel (13) ab der Satzgrenze der komplette weitere Äußerungsplan (semantisch, syntaktisch und pragmatisch) revidiert wurde, liegt in Beispiel (17) offensichtlich lediglich ein Umstieg von einer Versprachlichungsoption auf eine andere (unter Beibehaltung der semantischen und pragmatischen Disposition) vor: Die Zielkonstruktion Soviel vertraue [n habe ich dann doch nicht...] wird ersetzt durch So groß ist mein Vertrauen [...] dann doch nicht:

(17) $\mathrm{S}$ \{oviel vertraue $\{$ eviet-veratue $\}$ o groß ist mein Vertrauen $\}[\ldots]$ in $[\ldots]$ Leute, die ich noch nicht gesehen habe, dann doch nicht. .」)(BENSCH 14:18:28)

(Plan 1: Soviel vertrauen habe ich $\rightarrow$ Plan 2: So groß ist mein Vertrauen $)^{20}$

(18)aber wirklich günstig und \{ne riesen\{ne-riesen\}eine riesige\} Auswahl. (BENSCH 14:06:49)

(Plan 1: ne riesen Auswahl / Riesenauswahl $\rightarrow$ Plan 2: eine riesige Auswahl)

(19)@anew: Ich bin da eher pessimistisch... Ich will \{den Lasd\{den Easd\}Sachen notfalls zurück in den Laden bringen können\}, als sie per Post zu verschicken und zu hoffen, dass der Laden noch existiert... (BENSCH 14:15:32)

(Plan 1: Ich will den Laden notfalls aufouchen können, um die Sachen zurïckzubringen $\rightarrow$ Plan 2: Ich will die Sachen notfalls zurïck in den Laden bringen können)

(20) [bei teuren sachen] würde ich immer abholen, zumal man bei überweisung $\{$ ni $\{$ it $\}$ keine handhabe $\}$ hat (FALA 11:14:33)

(Plan 1: man ... nichts in der hand hat $\rightarrow$ Plan 2: man ... keine handhabe hat)

20 Bei den unter „Plan 1“ ausformulierten Strukturen handelt es sich um tentative Strukturen, die aus den eingegebenen Fragmenten unter Beibehaltung der propositionalen Struktur hätten entwickelt werden können; Tippfehler in den eingegebenen Texten (Lauifen/Kaufen, Lasd[en]/Laden) wurden bei der Formulierung der möglichen „Plan 1 "-Strukturen ignoriert. 
Zur Umplanung sind auch solche Fälle zu zählen, bei welchen der ursprünglich verfolgte Plan eine Fortführung der Struktur vorsah, mit der Revision aber der eingegebene Entwurf entgegen der Textverlaufsrichtung bis zu einem vorhandenen Abschlusspunkt gelöscht und der derart reduzierte Entwurf anschließend als Beitrag an den Server abgeschickt wird:

(21) Ich habe eBay früher (d.h. vor einigen Jahren) immer sehr gerne benutzt und war auch immer der Meinung, dass man dort durchaus "Schnäppchen" machen konnte. \{..aber \{.aber\}.」\} (BENSCH 13:45:09)

(22) Das verlief auch alles soweit OK\{, wie $\{-\ldots$ ie..$\rfloor\}$ (LARBE 16:14:15)

Die Reduktion einer geplanten Struktur muss nicht endgültig, sondern kann den gegebenen kommunikativen Rahmenbedingungen geschuldet sein. Im Beispiel (21) folgt der Verschickung unmittelbar eine weitere Produktionsphase nach, mit welcher ganz offensichtlich die ursprünglich vorgesehene Fortführung in Form einer eigenständigen syntaktischen Struktur (als neuer Beitrag) entworfen wird:

(23)Aber in letzter Zei[...]t [...] sind sämtliche Kategorien dermaßen von den sog. Powersellem bevölkert, die [...] hunderte gleiche Artikel anbieten, so dass es schwerfällt, das Gesuchte auch wirklich zu finden. $\downarrow$ (BENSCH $13: 45: 38)$

Die Revision in (21) dürfte somit darauf zurückzuführen sein, dass sich BENSCH während der Enkodierung kurzfristig dazu entschlossen hat, die Versprachlichung seines Handlungsplans in Anbetracht ihres Umfangs auf eine Sequenz aus zwei Beiträgen zu verteilen, anstatt sie in nur einem Beitrag (der dann, als Ganzer, erst deutlich später hätte verschickt werden können) zu realisieren (,Splitting'-Technik, vgl. Beißwenger 2007: 245-253 u. 261-264).

\section{d. Umstellung:}

Bei der Umstellung bezieht sich die Planänderung lediglich auf die Linearstruktur der geplanten Äußerung. In Beispiel (24) kann unter Einbeziehung des letzten vorausgegangenen Partnerbeitrags (im Beispiel in doppelten runden Klammern wiedergegeben) sowie der (anhand der Videobeobachtung rekonstruierbaren) Tatsache, dass dieser Partnerbeitrag von der Produzentin (INAB) vor Beginn ihrer Texteingabe auch tatsächlich wahrgenommen wurde, die Revision (sofern man we als Fragment des Adverbials wenn der mich zuvor ... reingelegt hat deutet) als Ergebnis einer Optimierung der Linearstruktur in Hinblick auf die thematische Organisation des Diskurses gedeutet werden: Die Ergänzungsfrage Wie wïrden Sie dem denn „Ärger“ machen? rhemati- 
siert mittels W-Deixis eine bestimmte Art des „Ärgermachens“. Da eine schlechte Bewertung abgeben den hierzu passenden rhematischen Ausdruck liefert, wenn der mich zuvor ... reingelegt hat aber nur eine für das Eintreten des rhematischen Sachverhalts geltende Einschränkung versprachlicht, rückt INAB den rhematischen Ausdruck ins Mittelfeld. Die Besetzung von Vorfeld und linker Verbklammer ist elliptisch (ich wïrde) und unter Einbeziehung der Frage rekonstruierbar:

(24)(( bsommer: Wie würden Sie dem denn „Ärger“ machen? )) \{we $\{w e\}$ eine schlechte bewertung abgeben, we\}nn der mich zuvor irgendwann mal [...] reingelegt hat (INAB 10:42:01)

\section{e. Komplexität:}

Manche Revisionen haben möglicherweise auch damit zu tun, dass die anvisierte Zielstruktur dem Produzierenden zu komplex wird. Die Wohlgeformtheitsanforderungen, die sich beim sukzessiven Formulieren etwa durch die Eröffnung einer komplexen Parenthese zwischen pränominalem Attribut und Nomen ergeben, wenn mit der betreffenden Phrase die übergeordnete syntaktische Struktur noch nicht zum Abschluss gebracht werden kann, können für die Verbalisierung unter Zeitdruck schnell zur Überforderung werden. In Bezug auf das folgende Beispiel könnte Komplexität eine plausible Erklärung für die Revision sein. Die Revision führt zu einer weniger komplexen Struktur, dafür aber auch zu einer weniger spezifischen Charakterisierung:

(25)Also ich würde grundsätzlich keine teure $\{n$ (d.h. für $\operatorname{mic}\{\mathrm{n}-(\mathrm{d}-\mathrm{h}-\mathrm{für}$ mie\}ren\} Artikel bei eBay kaufen. (BENSCH 14:18:11)

\subsubsection{Lexikalische Revisionen}

Als lexikalisch lassen sich solche Revisionen beschreiben, bei denen der revidierte und der ersetzende Ausdruck ohne wesentliche Bedeutungsveränderung paradigmatisch füreinander austauschbar sind. Die füreinander ausgetauschten Lexeme oder Konstruktionen unterscheiden sich z.B. stilistisch (etwa im Falle der Verben faken und türken), sind bedeutungsverwandt (etwa im Falle der Verben angeben und anzeigen) oder stellen dieselbe Sache oder Eigenschaft unter unterschiedlichem Aspekt dar (z.B. die Adjektive häufig und zunehmend, von denen ersteres einen bestimmten Frequenzgrad und zweiteres eine Art der Veränderung von Frequenz bezeichnet). Die lexikalische Ersetzung ist hierbei nicht durch den Redegegenstand, sondern stilistisch bedingt oder dient der Präzisierung. 
Beispiele für lexikalisch motivierte Ersetzungen sind:

(26) kommen $\rightarrow$ ansetzen: also muss ich generel in diesem Punkt nicht mit Misstrauen $\{\mathrm{ko}\{\mathrm{k} \Theta\}$ ansetzen $\}$ (MAGE 13:25:17)

(27) angeben $\rightarrow$ anzeigen:

im netz war eine glanzvolle verpackung ange\{gebe\{gehe\}zeigt\}, dabei war das spielt schon alt, aus den 80ern, als ausrede kam: "Oh, das war wohl ein Versehen". (MAGE 13:27:52)

(28) faken $\rightarrow$ tïrken:

Sag mal, ich glaube das war alles g\{efakt \{efakt\}etürkt\}, hier (INAB 10:56:34)

(29) filtern $\rightarrow$ ausfiltern:

[Warum eBay nicht einfach JavaScript in den Artikelbeschreibungen verbietet] \{und fi $\{\mathrm{fi}\}$ und ausfi\}ltert (!), verstehe ich nicht ganz. (BENSCH 13:52:41)

(30) dabei $\rightarrow$ genau da:

\{dabei \{dabei\}genau da\} setzt eine meiner fragen an: (MAGE 13:27:02)

(31) heute $\rightarrow$ später (Präzisierung):

Vielleicht schaue ich [...] mir die Seite \{gheute \{ später\} mal an, aber da ich eh keine [Anziehsachen bei eBay kaufe, ist das eher minderinteressant für mich.] (BENSCH 13:49:50)

An derselben Stelle einer aktuell in Versprachlichung befindlichen Struktur treten bisweilen nacheinander mehrere Revisionen unterschiedlichen Typs auf. Dies wirft ein Licht auf das „Verbalisierungslabor", das bei laufender Enkodierung noch mit dem Finden der geeigneten Struktur befasst ist und dabei u.U. auch mehrere Möglichkeiten ausprobiert:

(32) Revisionsschritt 1: Lexikalische Ersetzung (auf: Basis des Kontexts vermutbare Zielstruktur:

kommt es häufig vor, dass $\rightarrow$ kommt es zunehmend vor, dass): Aber in letzter Zeit kommt es \{häu\{hät\}zubne\} (BENSCH 13:45:38)

Revisionsschritt 2: Umplanung; lediglich das Temporaladverbial im Vorfeld wird beibehalten:

Aber in letzter Zei $\{t$ kommt es zubne \{tkemmt-es-zubne\}t sind sämtliche Kategorien dermaßen von den sog. Powersellern bevölkert, die [ .... hunderte gleiche Artikel anbieten\} (BENSCH 13:45:49) 


\subsubsection{Korrektur von Tippfehlern}

Revisionen zum Zweck der Korrektur von Tippfehlern stellen im untersuchten Korpus die zweitgrößte Gruppe unter den intrinsisch motivierten Revisionen. Typische Fälle sind solche, in denen anstelle der (dann/fann) oder zusätzlich zur anvisierten Taste (den/d3en) eine oder mehrere unmittelbar danebenliegende Tasten angeschlagen werden (Beispiele 83-35). Auch versehentliche Auslassungen kommen vor (Beispiel 36). Im Falle von „Buchstabendrehern“ (Beispiele 37-38) kommt vorübergehend die Linearisierung der Zeichenrealisierung durcheinander; typisch sind einfache Vertauschungen, bisweilen auch eine Kombination aus Verdrehern, einfachen Vertippern und der Auslassung von Zeichen („Buchstabensalat“, Beispiele 39-40).

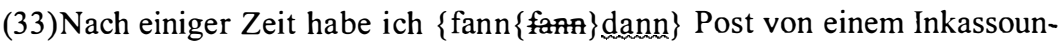
ternehmen bekommen. (LARBE 16:15:49)

(34)Was ist denn, wenn der Verkäufer sich weigert $\{d 3 e n\{d 3 e n\}$ den $\}$ artikel zurückzunehmen (INAB 10:52:07)

(35) (Wobei \{nabn\{aba\}man\} ja immer wieder von solchen Fällen hört.) (BENSCH 13:48:57)

(36)Das habe ich gar nicht so richtig mitbekom\{en, nu\{en, nu $\}$ men. Nu $\} \mathrm{r}$ mal am Rande gehört (bei heise.de), dass es da ein Problem gibt. (BENSCH 13:52:11)

(37)Direkt schlechte Erfahrungen nicht, außer, dass natürlich ein großer Teil

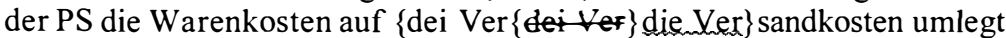
(was ich akzeptieren kann, oder halt auch nicht, dann kaufe ich einfach nicht)... (BENSCH 13:57:48)

(38)So ne richtige beratung ist das hier aber \{nciht \{ 11:01:18)

(39)es gibt \{sichr eihend \{s eihend $\}$ sicher reihen\} weise jugendliche, die dann auch ü (MAGE 13:45:47)

(40)Verzeichen Sie bitte meinen Sarkasmus, aber das läuft doch letztendlich darauf hinaus, dass $w$ \{ur ubn $\left\{\begin{array}{l}\text { ir }\} \\ {[\ldots]}\end{array}\right.$ (BENSCH 13:56:14)

\subsubsection{Orthographische Revisionen}

Einige Revisionen im untersuchten Korpus sind orthographisch motiviert. Der eigene Anspruch an die Normkonformität der schriftlichen Äußerung wird in diesen Fällen höher priorisiert als die Schnelligkeit der Produktion. Die Beispiele (41)-(45) zeigen vier Fälle der Korrektur 
von Klein- zu Großschreibung sowie einen Fall der Korrektur eines fehlenden Kommas in einer Aufzählung:

(41) Ah, \{sie \{sie\}Sie\} sind wieder da. (BENSCH 14:10:23)

(42)Wir waren bei den Powersellern, die man leider nicht ausfiltern kann \{und sie ha $\{$ sie-ha\} und Sie ha\}tten uns Suchtipps gegeben. (BENSCH $14: 11: 32)$

(43)@bsommer: Wenn man \{sie ni \{sieni\}Sie ni $\}$ cht filtern kann,war'sdas (BENSCH 14:12:32)

(44)[Mmh. Wahrsch]einlich haben Sie \{recht\{reeht\}Recht\}r (LARBE 16:35:20)

(45) [Verzeichen Sie bitte meinen Sarkasmus, aber das läuft doch letztentlich darauf hinaus,] dass man sich dann zig verschiedene Toolbars installieren würde: von eBay, von Google $\{$ von $\{\forall \Theta n\}$, von $\}$... (BENSCH 13:56:31)

\subsubsection{Morphologische Revisionen}

Zwei Revisionen im Korpus können als morphologisch beschrieben werden. Bei ihnen findet eine Kürzung der Formseite des revidierten Ausdrucks statt. Bei den ersetzenden Kurzformen (hab < habe, mal < einmal) handelt es sich um übliche und konventionalisierte, allerdings umgangssprachlich markierte Formvarianten. Interessant ist, dass diese nachträglichen morphologischen „Umkodierungen“ von Standard- zu Umgangssprache von demselben Chatter produziert wurden, der an anderer Stelle viermal durch nachträgliche orthographische Korrekturen auffällt (vgl. 3.2.4). In punkto Orthographie revidiert er mehrfach normorientiert, in den folgenden beiden Fällen weicht er hingegen nachträglich per Revision von der Norm ab:

(46) Ich hab $\{\mathrm{e}\{\mathbf{e}\}\}$ aber in den letzten 3 Monaten bestimmt $10[\ldots]$ Bücher bei Booklooker gekauft. (BENSCH 14:08:27)

(47) Wir haben uns schon \{einmal gegensei \{einmal-gegensei $\}$ mal gegensei\}tig beraten... :-) (BENSCH 14:10:34)

\subsubsection{Revision zum Zweck der Adressierung}

In drei Fällen wird dem Textentwurf per Revision eine Adressierung vorangestellt, mittels welcher explizit gemacht wird, dass sich der eigene Beitrag auf den (zuletzt gelesenen) Beitrag eines bestimmten Partners bezieht. Im Gegensatz zur extrinsisch motivierten Hinzufügung 
einer Adressierung in Beispiel (5) (Abschnitt 3.1) wird in diesen Fällen die Adressierung nicht aufgrund der Wahrnehmung eines oder mehrerer zwischenzeitlich am Bildschirm angezeigter Partnerbeiträge nachträglich eingefügt, sondern ohne Kenntnis neuer Partnerbeiträge, gewissermaßen prophylaktisch, gesetzt. Nachdem BENSCH in Beispiel (48) bereits das erste Zeichen seines intendierten Beitrags getippt hat, löscht er dieses noch einmal, um zuerst eine Adressierung $\mathrm{zu}$ realisieren und anschließend die Enkodierung seines Beitrags erneut zu beginnen:

(48) $\{\mathrm{D}\{\Theta\} @$ bsommer: $D\} . h$. sich [...] vorher zu Kategorien durchklicken und dann die [Suche in der jew. Kategorie durchführen.] (BENSCH $14: 16: 21)$

\subsubsection{Rein mentale Umplanungsaktivität}

In drei Fällen wird als ersetzender Ausdruck nach erfolgter Löschung der gelöschte Ausdruck erneut eingegeben. Solche Fälle werte ich als Anzeichen einer zurückgenommenen mentalen Umplanung, bei welcher zunächst vom ursprünglich verfolgten Plan abgerückt, anschließend aber sofort wieder zu diesem zurückgekehrt wird. Ein alternativer Plan ist noch nicht so weit konkretisiert, dass er bereits enkodierungsrelevant werden könnte und wird auch sofort wieder verworfen, um doch den ursprünglichen Plan wiederaufzugreifen. Da Planung und Enkodierung parallel laufen, wird das Abrücken vom Plan unmittelbar umgesetzt. Folglich erfordert die Rückkehr zum ursprünglichen Plan eine erneute Enkodierung des bereits Gelöschten:

(49) $\{\operatorname{Kos}\{$ Kes $\}$ Kosten $\}$ ? (LARBE 16:20:16)

(50) $\{$ Wenn $\{$ Wenn $\}$ Wenn $\}$ ich Mist baue, bin ich sicherlich bereit dafuer zu zahlen, aber (LARBE 16:21:58)

(51) Wenn ich Mist baue, bin ich sicherlich bereit dafuer zu zahlen\{, aber\{; abef $\}_{2}$ aber $\}$ wenn nicht, sollen die bitte die Kosten uebernehmen. (LARBE $16: 21: 58)$

\subsubsection{Gegenstandsbedingte Revisionen}

Gegenstandsbedingt sind solche Revisionen, die offensichtlich mit dem Redegegenstand und nicht mit dessen sprachlicher Repräsentation zu tun haben. In Beispiel (52) erinnert sich LARBE während der Enkodierung, dass er 300 anstatt 200 DM Gewinn gemacht hat, in Beispiel (53) 
daran, dass er als Käufer und nicht als Verkäufer gute Erfahrungen gemacht hat:

(52)Ja. Ich habe meine Sachen dort auch mir ca. 100-\{200\{z00\}300\} DM Gewinn versteigert. (LARBE 16:40:39)

(53)Nein. Bisher waren meine Erfahrungen mit anderen \{Ver\{ $\forall$ er $\}$ Kaeufern immer sehr gut. (LARBE 16:31:59)

\subsubsection{Graphische Hervorhebung}

In einem Fall dient die Revision der graphischen Hervorhebung. Im Beispiel

(54)[Warum eBay nicht einfach JavaScript in den Artikelbeschreibungen verbietet] \{und fi $\{\mathrm{f}\}$ und ausfi\}ltert (!), verstehe ich nicht ganz. (BENSCH 13:52:41)

wird nach der Löschung von und $f i$ die Konjunktion $u$ nd erneut eingegeben und mit einer netztypischen Hervorhebungsmarkierung (Unterstreichungen links und rechts) versehen. Des Weiteren findet im Beispiel eine lexikalische Ersetzung statt (filtern $\rightarrow$ ausfiltern).

\section{Bewertung der Ergebnisse und Ausblick}

Gegenüber mündlicher Kommunikation mit ihrer typischen (und zwingenden) Synchronisierung von Versprachlichung, Veräußerung und Wahrnehmung (,On line'-Zeitlichkeit) ist

schriftliche Verständigung [...] wesentlich die Herstellung und dann die Rezeption eines externen gegenständlichen Produkts, des Textes, der als solcher dauerhaft und zeitlich konstant ist. In der schriftlichen Verständigung steht das Produkt ,Text' im Vordergrund, nicht der Prozess, in dem er entsteht oder rezipiert wird. Ein vergleichbares Produkt gibt es im Prozess der mündlichen Verständigung nicht (Auer 2000: 61).

Das gilt für die Verbalisierung und Verarbeitung von Kommunikationsbeiträgen im Chat genauso wie in prototypisch monologischen Situationen der Textproduktion: Ausgetauscht werden Produkte sprachlicher Hervorbringung, auch wenn deren Wortlaut in aller Regel nicht auf eine dauerhafte Fixierung, sondern auf die unmittelbare Bearbeitung einer kommunikativen Aufgabe mit einem im zeitlichen Nahbereich verfügbaren Adressaten angelegt ist.

Diejenigen Phasen, in denen ein Chat-Beteiligter eigene Beiträge oder Beitragsteile produziert, ohne dabei den Stand des Bildschirmver- 
laufsprotokolls zu überprüfen, haben den Charakter kleiner monologischer Textproduktionsprozesse. Während der Produktion erarbeitet der Produzierende eine schriftliche Fassung dessen, was er an einem bestimmten Punkt seiner Kommunikationsteilnahme zum von ihm wahrgenommenen Stand des Kommunikationsgeschehens beitragen möchte - und zwar (so lange er nicht wieder auf den Bildschirm blickt) unabhängig davon, ob sich die Dokumentation des Kommunikationsgeschehens am Bildschirm in der Zwischenzeit verändert. Erst durch die Ausführung einer Verschickungshandlung wird der im Eingabeformularfeld stehende Text vom reversiblen Entwurf zu einem irreversiblen Produkt. Dieses kann dann für die Partner als Diskursbeitrag fungieren. Bis zur Verschickung kann der Entwurf beliebig geändert, umgeformt und ggf. auch wieder gelöscht werden.

Aufgrund des „Chatter-Dilemmas“, nicht gleichzeitig über einen längeren Zeitraum produzieren $u$ nd rezipieren zu können, zugleich aber sowohl produktiv als auch rezeptiv ,am Ball“ bleiben zu müssen, ergibt sich in Bezug auf die Produktion von Beitragstexten das Ideal, diese möglichst kurz zu halten und der Entscheidung zum sprachlichen Handeln möglichst rasch die Verschickung des versprachlichten Handlungszugs nachfolgen zu lassen. Entsprechend ist davon auszugehen, dass für die Konzeption der schriftlichen Beiträge nur geringe Planungsphasen in Anspruch genommen werden. Was Grabowski (1995) mit Blick auf prototypische Schreibprozesse und ihre Unterschiede zum Sprechen formuliert, dürfte im Chat daher gerade nicht gelten:

Zeiteinteilung: Beim kommunikativen Sprechen sind Menschen angehalten, keine zu langen Sprechpausen zu machen beziehungsweise Pausen durch turnerhaltende Signale zu füllen [...]. Der Sprecher muß also hinreichend schnell mit seiner Äußerung fortfahren. Es ist zu erwarten, daß diese Gewohnheit des Aufrechterhaltens einer gewissen Sprachproduktionsdichte auch beim dekontextualisierten Sprechen - wenn auch in abgeschwächter Form - wirksam wird. Schreiben ist dagegen, wenn kein Kommunikationspartner unmittelbar zugegen ist, ,self-paced“; der Schreibende kann die Enkodierung und graphomotorische Tätgikeit nach Belieben unterbrechen und seine kognitiven Ressourcen ausschließlich für sprachbezogene Planungsprozesse - u.a. für den Gedächtnisabruf - einsetzen, ohne mentale Energie darauf verwenden zu müssen, daß keine zu langen Pausen im Sprachproduktionsoutput entstehen (Grabowski 1995: 30).

Rau (1994) und Wrobel (1995) haben als Ergebnis von Think-aloudUntersuchungen $\mathrm{zu}$ Revisionen in handschriftlichen monologischen Textproduktionsprozessen herausgestellt, dass sich Formulieren zum großen Teil nicht in Form von Änderungen im niedergeschriebenen Text, sondern als mentale Versprachlichungsaktivität vollzieht. Wrobel geht davon aus, dass 
der Prozeß des Formulierens sich [...] nicht als „einfache“ Übersetzung mentaler Repräsentationen in sprachliche Äußerungen vollzieht, sondern vielmehr über mehr oder weniger sprachnahe Zwischenstufen, die sowohl mit vorgängigen abstrakten Prozessen der Textplanung als auch mit Prozessen des endgültigen Niederschreibens in Zusammenhang stehen (Wrobel 1995: 105f:).

Formulieren ist dabei vor allem Umformulieren bzw. ein Abwägen von Versprachlichungsalternativen. Die dabei tentativ gebildeten, noch nicht schriftlich enkodierten, aber sprachlich bereits weitgehend ausgeformten Vorstufen des zu produzierenden Textes bezeichnen Rau und Wrobel als Prätexte. Gegenüber ihren graphisch enkodierten Pendants sind Prätexte einfacher modifizierbar,

da sie den formalen Restriktionen der schriftlichen Sprache nicht bzw. nicht vollständig unterliegen. Sie ersparen damit einen Teil des kognitiven und aktionalen Aufwandes, der mit der Erzeugung und vor allem Veränderung manifester Textäußerungen verbunden ist (Wrobel 1995: 115). ${ }^{21}$

Für unter monologischen Bedingungen, „self-paced“ (Grabowski), vollzogene Schreibprozesse ist nach Wrobel (1995) davon auszugehen, dass das Formulieren dem Enkodieren vorausgeht (daher auch der Terminus ,Prätext'):

Formulierungen als Endprodukte des Schreibproduktionsprozesses werden [...] nicht im Prozeß des Niederschreibens erzeugt, verändert oder ,verbessert“. Sie liegen vielmehr am ,point of inscription“ bereits vor (Wrobel 1995: $101)$.

Chat-Beiträge sind im Gegensatz zu monologisch verfassten Texten nicht für die situationsentbundene Rezeption, sondern für die Bearbeitung einer aktuellen kommunikativen Aufgabe konzipiert. Sie fungieren als kontextuell determinierte Teile einer dialogisch realisierten Handlungssequenz; Handlungsverkettungen innerhalb desselben Beitrags sind hingegen selten. Anforderungen, die sich aus gewählten Textmustern und dem Ideal maximaler und situationsunabhängiger Verständlichkeit ergeben, spielen somit beim Formulieren im Chat keine Rolle. Stattdessen steht die Notwendigkeit im Vordergrund, ab dem Zeitpunkt, zu welchem ein Handlungsplan gefasst wird, für diesen möglichst rasch

21 Hierzu ist anzumerken, dass der Aufwandsunterschied beim Korrigieren von Texten gegenüber Prätexten in handschriftlichen Schreibprozessen (bei denen im Zuge der Korrektur ein Durchstreichen, häufig ein Über- oder Unter-die-Zeilen-Schreiben erforderlich sowie generell der für Korrekturen zur Verfügung stehende Raum auf der Schreibfläche begrenzt ist) höher ist als beim Schreiben am Computer, da sich Getipptes „leichter löschen, fortwährend ändern, umstellen, kopieren, verknüpfen und überarbeiten“" lässt (Schmitz 2003: 256f.). 
eine angemessene sprachliche Form zu finden, diese möglichst zügig schriftlich $\mathrm{zu}$ enkodieren und $\mathrm{zu}$ verschicken. Dabei spielen u.a. der gewählte Handlungstyp (z.B. Assertion), die Position im Handlungsmuster, die mit dem Beitrag realisiert werden soll (z.B. Antwort auf Frage), die in der Vorkommunikation bereits eingeführten Redegegenstände, die soziale Beziehung zwischen den Beteiligten sowie der Grad der Orientierung an der schriftsprachlichen Norm eine Rolle. Revisionen, sofern nicht unbedingt erforderlich, beeinträchtigen die zügige Bearbeitung der Versprachlichungsaufgabe. Dennoch sind Revisionen bei der Produktion von Chat-Beiträgen keine Seltenheit. Im Falle intrinsisch motivierter Revisionen kann als Grund der Verzicht auf Planungszeiten und das Parallellaufen von Formulieren und Enkodieren vermutet, im Falle extrinsisch motivierter Revisionen die Anpassung eines aktuell versprachlichten Handlungsplans an veränderte Kontextbedingungen angenommen werden:

- Die Ergebnisse der in Abschnitt 3.2 beschriebenen explorativen Untersuchung zu intrinsisch motivierten Revisionen beim Chatten legen nahe, dass bei der Verbalisierung im Chat die Prätextbildung (das tentative Ausprobieren von Formulierungsvarianten) und die schriftliche Enkodierung nicht sukzessive erfolgen, sondern dass der Formulierungs- und der Enkodierungsprozess simultan ineinandergreifen: Während eine Einheit noch in Ausformulierung begriffen ist, wird sie bereits schriftlich enkodiert. Kommt es zu Unsicherheiten in Bezug auf die sprachliche Form (u.U. ausgelöst durch das Mitlesen und Evaluieren des beim Tippen im Eingabeformularfeld entstehenden Textentwurfs), wird im Bedarfsfall direkt reformuliert. Die digitale Schriftlichkeit mit ihrer Möglichkeit zur spurlosen Beseitigung einmal eingegebener Zeichen unterstützt diese Form des Formulierens im Enkodierungsprozess (anstatt in einem separaten Teilprozess des Schreibprozesses) in hervorragender Weise. Ein Indiz hierfür ist, dass die größte Gruppe der Revisionen (57 von 122 Fällen) nicht auf die Beseitigung von Tippfehlern gerichtet ist, sondern im weitesten Sinne als syntaktisch motiviert gelten kann. Insgesamt machen Tippfehler nur etwa ein Drittel (42 von 122 Fällen) der im untersuchten Korpus vorgefundenen Revisionsanlässe aus.

- Die Ergebnisse der in Abschnitt 3.1 beschriebenen Untersuchung zu extrinsisch motivierten Revisionen legen nahe, dass Revisionen auch für die Kohärenzplanung beim Chatten als eine zentrale Ressource genutzt werden. Wo ein Turn-taking-Management zur Laufzeit nicht als Strukturierungsinstrument zur Verfügung steht, son- 
dern jeder Beteiligte jederzeit mit der Produktion eines neuen Beitrags beginnen kann und das Eintreffen neuer Beiträge am Bildschirm nicht unbedingt immer sofort von den Partnern bemerkt wird, ist es Sache des einzelnen Teilnehmers, sein Handeln immer wieder neu an unvorhergesehene Veränderungen im Stand des Kommunikationsverlaufs anzupassen. Die vollständige Löschung, Umplanung und Neuproduktion ist eine häufig genutzte Strategie, mit dieser Anforderung umzugehen.

Abschließend ist festzuhalten, dass Einblicknahme in den Prozess der Beitragsproduktion beim Chatten Hinweise darauf liefert, dass die Persistenzeigenschaften des graphischen Mediums von den ChatBeteiligten als Ressource genutzt werden, um mit der fehlenden Möglichkeit zur Synchronisierung von Hervorbringung und Verarbeitung umzugehen und die somit fehlende Möglichkeit einer interpersonalen Handlungskoordination zur Laufzeit zu kompensieren. Auch der Tatsache, dass schriftliche Enkodierung aufwändiger ist als mündliche, wird durch die systematische Nutzung der Persistenzeigenschaften des Mediums begegnet: $\mathrm{Da}$ eingegebener Text gegebenenfalls jederzeit revidiert werden kann, kann auf im Verbalisierungsprozess vorgeschaltete Formulierungsphasen verzichtet und direkt mit der Enkodierung geplanter Beiträge begonnen werden.

Da fehlende Synchronisierung und erhöhter Produktionsaufwand wiederum durch die materialen Eigenschaften des graphischen Mediums bedingt sind, wirkt sich das Distanzmedium Schrift in doppelter Weise auf den Austausch aus: einerseits als Faktor, der die Annäherung des Chat an den mündlichen Diskurs in starker Weise limitiert (von allen bekannten Diskursformen ist der Chat diejenige, die sich strukturell am deutlichsten vom elementaren Diskurs unterscheidet), andererseits als Ressource für die Nutzung von produktiven Möglichkeiten (insbesondere der Möglichkeit zur Textrevision), die im Diskurs ansonsten nicht zur Verfügung stehen.

Weitere Untersuchungen zu Strategien des Umgangs der Chatter mit der Chat-Situation auf Basis von Nutzerbeobachtungen versprechen Gewinn. Ein umfassendes Verständnis der Chat-Kommunikation, insbesondere in Hinblick auf ihre Stellung zu traditionellen Formen der Nähe- bzw. Distanzkommunikation, ist derzeit m.E. noch nicht erreicht. Der Weg dahin bleibt spannend. 


\section{Literatur}

Ágel, Vilmos/Hennig, Mathilde (2007): Überlegungen zur Theorie und Praxis des Nähe- und Distanzsprechens. In: Ágel, Vilmos/Hennig, Mathilde (Hrsg.): Zugänge zur Grammatik der gesprochenen Sprache. Tübingen: Niemeyer (Reihe Germanistische Linguistik 269), 179-214.

Auer, Peter (2000): On line-Syntax - oder: was es bedeuten könnte, die Zeitlichkeit der mündlichen Sprache ernst zu nehmen. In: Sprache und Literatur 85, $43-56$.

Beißwenger, Michael (Hrsg.) (2001): Chat-Kommunikation. Sprache, Interaktion, Sozialität \& Identität in synchroner computervermittelter Kommunikation. Perspektiven auf ein interdisziplinäres Forschungsfeld. Stuttgart: ibidem.

Beißwenger, Michael (2002): Getippte „Gespräche“ und ihre trägermediale Bedingtheit. Zum Einfluß technischer und prozeduraler Faktoren auf die kommunikative Grundhaltung beim Chatten. In: Schröder, Ingo W./Voell, Stéphane (Hrsg.): Moderne Oralität. Marburg: Förderverein Völkerkunde in Marburg (Reihe Curupira), 265-299.

Beißwenger, Michael (2003): Sprachhandlungskoordination im Chat. In: Zeitschrift für germanistische Linguistik 31, 198-231.

Beißwenger, Michael (2007): Sprachhandlungskoordination in der Chat-Kommunikation. Berlin/New York: de Gruyter (Linguistik - Impulse \& Tendenzen 26).

Bittner, Johannes (2003): Digitalität, Sprache, Kommunikation. Eine Untersuchung zur Medialität von digitalen Kommunikationsformen und Textsorten und deren varietätenlinguistischer Modellierung. Berlin: Erich Schmidt (Philologische Studien und Quellen 178).

Crystal, David (2001): Language and the Internet. Cambridge: Cambridge University Press.

Dürscheid, Christa (2004): Netzsprache - ein neuer Mythos. In: Beißwenger, Michael/Hoffmann, Ludger/Storrer, Angelika (Hrsg.): Internetbasierte Kommunikation. Duisburg: Red. OBST (Osnabrücker Beiträge zur Sprachtheorie 68), 141-157.

Dürscheid, Christa (2005): Medien, Kommunikationsformen, kommunikative Gattungen. In: Linguistik online 22. http://www.linguistik-online.de/22_05/ duerscheid.pdf (Stand: 06.12.2008).

Dürscheid, Christa (2006): Einführung in die Schriftlinguistik. 3., überarbeitete und ergänzte Auflage. Göttingen: Vandenhoeck \& Ruprecht (Studienbücher zur Linguistik 8).

Ehlich, Konrad (1983): Text und sprachliches Handeln. Die Entstehung von Texten aus dem Bedürfnis nach Überlieferung. In: Assmann, Aleida/Assmann, Jan et al. (Hrsg.): Schrift und Gedächtnis. Archäologie der literarischen Kommunikation I. München: Fink, 24-43.

Ehlich, Konrad (1984): Zum Textbegriff: In: Rothkegel, Annely/Sandig, Barbara (Hrsg.): Text - Textsorten - Semantik. Hamburg: Buske, 9-25.

Ehlich, Konrad (1989): Zur Genese von Textformen. In: Antos, Gerd/Krings, Hans-Peter (Hrsg.): Textproduktion. Tübingen: Niemeyer, 84-99.

Ehlich, Konrad (1994): Funktion und Struktur schriftlicher Kommunikation In: Günther, Hartmut/Ludwig, Otto (Hrsg.): Schrift und Schriftlichkeit. Writing 
and Its Use. Ein interdisziplinäres Handbuch internationaler Forschung. 1. Halbbd. Berlin/New York: de Gruyter (Handbücher zur Sprach- und Kommunikationswissenschaft 12.1), 18-41.

Ehlich, Konrad/Rehbein, Jochen (1986): Muster und Institution. Untersuchungen zur schulischen Kommunikation. Tübingen: Narr (Kommunikation und Institution 15).

Garcia, Angela C./Jacobs, Jennifer Baker (1998): The Interactional Organization of Computer Mediated Communication in the College Classroom. In: Qualitative Sociology 21, 299-317.

Garcia, Angela C./Jacobs, Jennifer Baker (1999): The Eyes of the Beholder: Understanding the Turn-Taking System in Quasi-Synchronous ComputerMediated Communication. In: Research on Language and Social Interaction $32,337-367$.

Grabowski, Joachim (1995): Schreiben als Systemregulation. Ansätze einer psychologischen Theorie der schriftlichen Sprachproduktion. In: Jakobs, EvaMaria/Knorr, Dagmar/Molitor-Lübbert, Sylvie (Hrsg.): Wissenschaftliche Textproduktion. Mit und ohne Computer. Frankfurt a.M. et al.: Lang, 11-34.

Hennig, Mathilde (2001): Das Phänomen des Chat. In: Jahrbuch der Ungarischen Germanistik, 215-239.

Herrmann, Theo/Grabowski, Joachim (1994): Sprechen. Psychologie der Sprachproduktion. Heidelberg/Berlin/Oxford: Springer.

Hoffmann, Ludger (2004): Chat und Thema. In: Beißwenger, Michael/Hoffmann, Ludger/Storrer, Angelika (Hrsg.): Internetbasierte Kommunikation (Osnabrücker Beiträge zur Sprachtheorie 68), 103-122. [Ergänzte Version als OnlineRessource unter http://home.edo.uni-dortmund.de/ hoffmann/PDF/Chat_Themal.pdf (Stand: 06.12.2008)].

Jones, Rodney (2001): Beyond the Screen. A Participatory Study of Computer Mediated Communication Among Hong Kong Youth. Paper presented at the Annual Meeting of the American Anthropological Association Nov. 28-Dec. 2, 2001. http://personal.cityu. edu.hk/ enrodney/Research/ICQPaper.doc (Stand: 06.12.2008).

Klein, Wolfgang (1985): Gesprochene Sprache - geschriebene Sprache. In: Zeitschrift für Literaturwissenschaft und Linguistik 59, 9-35.

Koch, Peter/Oesterreicher, Wulf (1985): Sprache der Nähe - Sprache der Distanz. Mündlichkeit und Schriftlichkeit im Spannungsfeld von Sprachtheorie und Sprachgeschichte. In: Romanistisches Jahrbuch 36, 15-43.

Koch, Peter/Oesterreicher, Wulf (1994): Schriftlichkeit und Sprache. In: Günther, Hartmut/Ludwig, Otto (Hrsg.): Schrift und Schriftlichkeit. Writing and Its Use. Ein interdisziplinäres Handbuch internationaler Forschung. 1. Halbbd. Berlin/New York (Handbücher zur Sprach- und Kommunikationswissenschaft 12.1), 587-604.

Koch, Peter/Oesterreicher, Wulf (2007): Schriftlichkeit und kommunikative Distanz. In: Zeitschrift für germanistische Linguistik 35, 346-375.

Lemnitzer, Lothar/Naumann, Karin (2001): „Auf Wiederlesen!“ - das schriftlich verfasste Unterrichtsgespräch in der computervermittelten Kommunikation. Bericht von einem virtuellen Seminar. In: Beißwenger, Michael (Hrsg.): ChatKommunikation. Sprache, Interaktion, Sozialiät \& Identität in synchroner 
computervermittelter Kommunikation. Perspektiven auf ein interdisziplinäres Forschungsfeld. Stuttgart: ibidem, 469-491.

Marcoccia, Michel/Atifi, Hassan/Gauducheau, Nadia (2008): Text-centered versus multimodal analysis of Instant Messaging conversation. In: Androutsopoulos, Jannis/Beißwenger, Michael (Hrsg.): Data and Methods in ComputerMediated Discourse Analysis. Special issue of Language@Internet. http:// www.languageatinternet.de/articles/2008/1621/ (Stand: 06.12.2008).

Markman, Kris (2006): Computer-Mediated Conversation: The Organization of Talk in Chat-Based Virtual Team Meetings. Dissertation, University Texas at Austin. [Abstract unter http://linguistlist.org/issues/17/17-1709.html (Stand: 06.12.2008)].

Meise-Kuhn, Katrin (1998): Zwischen Mündlichkeit und Schriftlichkeit. In: Brock, Alexander/Hartung, Martin (Hrsg.): Neuere Entwicklungen in der Gesprächsforschung. Tübingen: Narr (ScriptOralia 108), 213-235.

Ogura, Kanayo/Nishimoto, Kazushi (2004): Is a Face-to-Face Conversation Model Applicable to Chat Conversations? Paper presented at the Eighth Pacific Rim International Conference on Artificial Intelligence (PRICAI2004). http://ultimavi.arc.net.my/banana/Workshop/PRICAI2004/Final/ogura.pdf (Stand: 06. 12.2008).

Rau, Cornelia (1994): Revisionen beim Schreiben. Zur Bedeutung von Veränderungen in Textproduktionsprozessen. Tübingen: Niemeyer (Reihe Germanistische Linguistik 148).

Rintel, E. Sean/Pittam, Jeffirey (1997): Strangers in a Strange Land. Interaction Management on Internet Relay Chat. In: Human Communication Research 23, 507-534.

Runkehl, Jens/Schlobinski, Peter/Siever, Torsten (1998): Sprache und Kommunikation im Internet. Überblick und Analysen. Opladen/Wiesbaden: Westdeutscher Verlag.

Schmitz, Ulrich (2003): Schreiben und neue Medien. In: Bredel, Ursula/Günther, Hartmut/Klotz, Peter/Ossner, Jakob/Siebert-Ott, Gesa (Hrsg.): Didaktik der deutschen Sprache. Ein Handbuch. 1. Teilband. Paderborn: Schöningh, 249260.

Spitzmüller, Jürgen (2005): Spricht da jemand? Konzeption und Repräsentation in virtuellen Räumen. In: Kramorenko, Galina (Hrsg.): Aktual'nije problemi germanistiki i romanistiki [Aktuelle Probleme der Germanistik und der Romanistik], Bd. 9, Teil I: Slovo v jasyke u retschi [Das Wort in Sprache und Rede]. Smolensk: Smolensk State Pedagogical University, 33-56. Preprint: http://www.ds.unizh.ch/lehrstuhlduerscheid/docs/spitzm/chat-05.pdf (Stand: 06.12.2008).

Storrer, Angelika (2001): Getippte Gespräche oder dialogische Texte? Zur kommunikationstheoretischen Einordnung der Chat-Kommunikation. In: Lehr, Andrea/Kammerer, Matthias/Konerding, Klaus-Peter/Storrer, Angelika, et al. (Hrsg.): Sprache im Alltag. Beiträge zu neuen Perspektiven in der Linguistik. Herbert Ernst Wiegand zum 65. Geburtstag gewidmet. Berlin/New York: de Gruyter, 439-465.

Storrer, Angelika (2009, i.Dr.): Rhetorisch-stilistische Eigenschaften der Sprache des Internets. In: Fix, Ulla/Gardt, Andreas/Knape, Joachim (Hrsg.): Rhetorik und Stilistik. Rhetorics and Stilistics. Ein internationales Handbuch histori- 
scher und systematischer Forschung. Berlin/New York: de Gruyter (Handbücher zur Sprach- und Kommunikationswissenschaft 31).

Tannen, Deborah (1984): Spoken and written narratives in English and Greek. In: Deborah Tannen (Hrsg.): Coherence in spoken and written discourse. Norwood: Ablex (Advances in Discourse Processes 12), 21-41.

Vronay, David/Smith, Marc/Drucker, Steven (1999): Alternative interfaces for chat. In: Proceedings of the 12th annual ACM symposium on User interface software and technology (CHI Letters 1,1), 19-26.

Wichter, Sigurd (1991): Zur Computerwortschatz-Ausbreitung in die Gemeinsprache. Elemente der vertikalen Sprachgeschichte einer Sache. Frankfurt a.M. et al.: Lang (Germanistische Arbeiten zu Sprache und Kulturgeschichte 17).

Wrobel, Arne (1995): Schreiben als Handlung. Überlegungen und Untersuchungen zur Theorie der Textproduktion. Tübingen: Niemeyer (Reihe Germanistische Linguistik 158).

Zifonun, Gisela/Hoffmann, Ludger/Strecker, Bruno (1997): Grammatik der deutschen Sprache. 3 Bde. Berlin/New York: de Gruyter (Schriften des Instituts für deutsche Sprache 7.1-7.3). 
Dieser Text wird über DuEPublico, dem Dokumenten- und Publikationsserver der Universität Duisburg-Essen, zur Verfügung gestellt. Die hier veröffentlichte Version der E-Publikation kann von einer eventuell ebenfalls veröffentlichten Verlagsversion abweichen.

DOI: $10.1515 / 9783110220872.247$

URN: urn:nbn:de:hbz:464-20201201-122146-3

Beißwenger, Michael: Chattern unter die Finger geschaut: Formulieren und Revidieren bei der schriftlichen Verbalisierung in synchroner internetbasierter Kommunikation.

In: Nähe und Distanz im Kontext variationslinguistischer Forschung / Ágel, Vilmos ;

Hennig, Mathilde (Hrsg.). Berlin [u.a.]: De Gruyter, 2010. S. 247-294

e-ISBN 978-3-11-022087-2. DOI: https://doi.org/10.1515/9783110220872.

(C) 2010 Walter de Gruyter Alle Rechte vorbehalten. 